\title{
Teoría prospectiva: un análisis de la decisión bajo riesgo*
}

\section{Daniel Kabneman y Amos Tversky ${ }^{1}$}

\begin{abstract}
Este artículo presenta una crítica a la teoría de la utilidad esperada como modelo descriptivo de la toma de decisiones bajo riesgo y presenta un modelo alternativo llamado teoría prospectiva. Las elecciones entre alternativas arriesgadas muestran diversos efectos generales que son inconsistentes con los principios básicos de la teoría de utilidad. En concreto, la gente tiende a ponderar menos los resultados que son solamente probables en comparación con los resultados que se obtienen con seguridad. Esta tendencia, a la que llamamos el efecto de certidumbre, contribuye a la aversión por el riesgo cuando se tratan de ganancias seguras y a la atracción por el riesgo en el caso de elecciones con pérdidas seguras. Además, la gente, generalmente, descarta aquellos componentes que son iguales en todas las alternativas que se están considerando. Esta tendencia, llamada efecto de aislamiento, lleva a preferencias inconsistentes cuando una misma elección se presenta de formas diferentes. Se desarrolla una teoría alternativa de la elección, donde los valores de medida son asignados a las ganancias y a las pérdidas en vez de a los resultados finales y donde se sustituyen las probabilidades por pesos de decisión. La función de valoración es normalmente cóncava para las ganancias y normalmente convexa para las pérdidas, y generalamente más acelerada para las pérdidas que para las ganancias. Los pesos de decisión son, generalmente, más bajos que sus correspondientes probabilidades, excepto en el caso de probabilidades bajas. Que se ponderen más las probabilidades bajas puede contribuir a la atracción tanto por el juego como por la compra de seguros.
\end{abstract}

\section{INTRODUCCION}

La teoría de la utilidad esperada ha dominado el análisis de la toma de decisiones bajo riesgo. Generalmente, esta teoría se ha aceptado como un modelo normativo de la elección racional (24), y como modelo descriptivo ha sido aplicada ampliamente a la conducta económica, por ejemplo $(15,4)$. De esta manera, se ha considerado que cualquier persona sensata preferiría atenerse a los axiomas de la teoría $(47,36)$ la mayoría de las veces, que es lo que hacen la mayor parte de las personas en realidad.

El presente artículo describe distintas clases de problemas de elección donde las preferencias de los sujetos violan sistemáticamente los axiomas de la teoría de la utilidad esperada. A la luz de estas observaciones, mantenemos que la teoría de la utilidad, tal y como normal-

* «Prospect Theory: An Analysis of Decision under Risk», en Econometrica, vol. 47, marzo de 1979, n. ${ }^{2}$ 2, págs. 263-291. Traducción castelana por Hilda Gambara D’Errico.

1. Parte de este trabajo se ha realizado a través de las becas de la fundación Harry F. Guggenheim y del «Advanced Research Projects Agency» del Departamento de Defensa y supervisadas por la «Oficina de investigación Naval» bajo el contrato N00014-78-C-0100 (ARPA Order No. 3469) bajo el subcontrato 78-072-0722 de «Decisions and Designs. Inc» a «Perceptronics Inc.». También agradecemos al «Centro para estudios avanzados en Ciencias de la Conducta» de Stanford el apoyo prestado. 
mente se ha interpretado y aplicado, no es un modelo descriptivo adecuado; proponemos un enfoque alternativo para el estudio de la elección bajo riesgo.

\section{CRITICAS}

La toma de decisiones bajo riesgo puede considerarse como una elección entre alternativas o jugadas. Una alternativa $(\mathrm{X} 1, \mathrm{P} 1 ; \ldots ; \mathrm{XmPn})$ es una relación que mantiene el resultado $\mathrm{Xi}$ con la probabilidad $\mathrm{Pi}$, donde $\mathrm{P} 1+\mathrm{P} 2+\ldots+\mathrm{Pn}=1$. Para simplificar la notación, omitimos los resultados nulos y usamos $(\mathrm{X}, \mathrm{P})$ para denotar la alternativa $(\mathrm{X}, \mathrm{P} ; \mathrm{O}, 1-\mathrm{P})$ que presenta $\mathrm{X}$ con probabilidad $\mathrm{P}$ y $\mathrm{O}$ con probabilidad $1-\mathrm{P}$. La alternativa ( $\sin$ riesgo) que presenta $\mathrm{X}$ con seguridad se denota por $(\mathrm{X})$. La presente discusión se restringe a aquellas alternativas con probabilidades objetivas.

La aplicación de la teoría de utilidad esperada para las elecciones entre alternativas se basa en los tres principios siguientes:

(i) Esperanza: $\mathrm{U}(\mathrm{X} 1, \mathrm{P} 1 ; \ldots ; \mathrm{Xn}, \mathrm{Pn})=\mathrm{P} 1 \mathrm{U}(\mathrm{X} 1)+\ldots+\mathrm{PnU}(\mathrm{Xn})$.

Esto es, la utilidad total de una alternativa, denotada por $\mathrm{U}$, es la utilidad esperada de sus resultados.

(ii) Integración de valores: $(\mathrm{X} 1, \mathrm{P} 1 ; \ldots \mathrm{Xn}, \mathrm{Pn})$ se acepta respecto de un valor cualquiera de medida $\mathrm{W}$ si $\mathrm{U}(\mathrm{W}+\mathrm{X} 1, \mathrm{P} 1 ; \ldots ; \mathrm{W}+\mathrm{Xn}, \mathrm{Pn})>\mathrm{U}(\mathrm{W})$.

Esto es, una alternativa se acepta respecto de un valor si la utilidad resultante de agregar en la propia alternativa el valor dado es superior a la utilidad del valor aislado. Así, el dominio de la función de utilidad son las utilidades finales (que incluyen el valor de medida) más que las diferencias positivas o negativas respecto del valor de medida.

A pesar de que el dominio de la función de utilidad no se limita a ninguna clase particular de consecuencias, la mayoría de las aplicaciones de la teoría se han dirigido a resultados monetarios. Además, la mayoría de las aplicaciones económicas introducen el siguiente supuesto adicional.

(iii) Aversión al riesgo: u es cóncava (u"<0).

Una persona tiene aversión por el riesgo si prefiere una determinada alternativa ( $x$ ) a cualquier otra alternativa arriesgada cuyo valor esperado sea $x$. En la teoría de la utilidad esperada, la aversión por el riesgo es equivalente a la concavidad de la función de utilidad. El predominio de la aversión por el riesgo es, quizá, la generalización más conocida respecto a las elecciones arriesgadas. Esto llevó a que los últimos investigadores sobre la decisión de la década de los ochenta propusieran una función de utilidad cóncava para el dinero, esta idea se ha seguido manteniendo en trabajos posteriores (Pratt, 33, Arrow, 4).

En las secciones siguientes demostramos diversos fenómenos que violan estos principios de la teoría de utilidad esperada. Las demostraciones están basadas en las respuestas de estudiantes de universidad sobre problemas de elección hipotéticos. A los sujetos se les presentaron problemas del tipo que se ejemplifica a continuación.

¿Cuál de las siguientes opciones preferiría?

A: 50 por 100 de posibilidades de ganar 1000 .

50 por 100 de posibilidades de no ganar nada.

B: 450 seguras.

Los resultados se referían a dinero israelita. Para apreciar la significación de las cantidades presentadas, nótese que el ingreso. medio 
mensual de una familia es aproximadamente 3000 libras israelitas. A los sujetos se les pidió que imaginaran que realmente se presentaban al problema de elección y que especificaran por cuál de las opciones se decidirían. Las respuestas fueron anónimas y en las instrucciones se les especificó que no había ninguna respuesta «correcta» para este tipo de problemas y que la finalidad del estudio era averiguar cómo la gente elegía entre alternativas arriesgadas. Los problemas se presentaron en forma de cuestionario, con doce problemas por cuadernillo. Se construyeron distintas formas de cada cuestionario, de tal manera que a los sujetos se les presentaron los problemas en diferentes órdenes. Además, se usaron dos versiones de cada problema donde se invertía la posición derecha-izquierda de la alternativa.

Los problemas que se describen en este artículo se han seleccionado para demostrar una serie de efectos. Cada efecto se ha observado en diversos problemas con distintos resultados y probabilidades. Alguno de los problemas se presentaron también a grupos de estudiantes de la universidad de Estocolmo y de Michigan. En general los resultados fueron esencialmente idénticos a los encontrados con los sujetos israelíes.

El procedimiento de las elecciones hipotéticas nos lleva a la formulación de cuestiones referentes a la validez del método y a la generalización de los resultados. Nosotros somos conscientes de estos problemas. Sin embargo, todos los métodos que se han utilizado para probar la teoría de la utilidad sufren, también, de importantes desventajas. Las elecciones reales pueden ser investigadas tanto en el ambiente real, por observaciones estadísticas de la conducta económica, como en el laboratorio. Los estudios de campo sólo pueden ser útiles para hacer comprobaciones bastante groseras de predicciones cualitativas; ya que las probabilidades y las utilidades no pueden ser medidas de una manera adecuada en tales contextos. Los experimentos de laboratorio se han diseñado con el fin de obtener medidas precisas de utilidad y probabilidad de las elecciones reales, sin embargo, estos estudios experimentales generalmente implican juegos ingeniosos con apuestas pequeñas, y un gran número de repeticiones de problemas muy parecidos. Estas características de las tareas de laboratorio complican la interpretación de los resultados y restringen su generalización.

Al menos, el método de elecciones hipotéticas aparece como un procedimiento más sencillo a través del cual se pueden investigar un gran número de cuestiones teóricas. El uso de este método descansa en el supuesto de que la gente, a menudo, sabe cómo comportarse en situaciones reales de elección, y en el supuesto de que los sujetos no tienen ninguna razón especial para hacerlo de manera distinta, en la situación experimental. Si la gente predice sus elecciones con cierta exactitud, la existencia de violaciones sistemáticas de la teoría de la utilidad esperada con problemas hipotéticos iría en contra de esta teoría.

\section{Certeza, probabilidad y posibilidad}

En la teoría de la utilidad esperada, las utilidades de los resultados se ponderan por sus probabilidades. En la presente sección se describe una serie de problemas de elección donde las preferencias de los sujetos violan de una manera sistemática este principio. Primeramente demostramos que la gente pondera más los resultados que considera con 
certeza, que aquellos resultados que son solamente probables; a este efecto lo hemos denominado efecto de certidumbre.

El contraejemplo que se conoce mejor y que muestra el efecto de certeza fue el introducido por el economista francés Maurice Allais en 1953. El ejemplo propuesto por Allais ha sido objeto de discusión por diversos autores, tanto desde un punto de vista descriptivo como normativo. Los dos tipos de problemas que presentamos a continuación son variaciones del ejemplo de Allais, sin embargo difieren de éste en que las ganancias que se presentan pueden considerarse más bien moderadas y no grandes cantidades. El número de sujetos que contestó cada problema se denota con $\mathrm{N}$, y el porcentaje de éstos que eligieron cada opción se eșpecifica entre paréntesis.

Problema 1: Elegir entre

A: 2500 con probabilidad 33

2400 con probabilidad 66

0 con probabilidad .01

B: 2400 seguras

$\mathrm{N}=72$ (18) para $A$

$(82) *$ para B

Problema 2: Elegir entre

C: 2500 con probabilidad .33

0 con probabilidad .67

D: 2400 con probabilidad .34

0 con probabilidad .66

$\mathrm{N}=72 \quad(83) *$ para $\mathrm{C}$

(17) para $\mathrm{D}$

Como muestran los datos el 82 por 100 de los sujetos eligieron B en el problema 1, y el 83 por 100 de los sujetos eligieron C en el problema 2. Cada una de estas diferencias es significativa con un nivel de .01 (se denota con el asterisco). Además, el análisis individual de las elecciones indica que la mayoría de los sujetos (61 por 100) hicieron la elección modal en ambos problemas. Este patrón de preferencias, en la manera que fue descrito originalmente por Allais, viola la teoría de la utilidad esperada. Según esta teoría, con $u(0)=0$, la preferencia primera implica

$$
\begin{aligned}
& u(2400)>.33 u(2500)+.66 u(2400) \text { ó } \\
& .34 u(2400)>.33 u(2500)
\end{aligned}
$$

Mientras que la segunda preferencia implica la desigualdad inversa. Fijese que el problema 2 se ha obtenido del problema 1 por la eliminación de la probabilidad .66 de ganar 2400 de ambas alternativas. Evidentemente, este cambio produce una mayor reducción en deseabilidad cuando se cambia el carácter de una alternativa que antes era segura ahora es probable, que cuando la alternativa original y la modificada son inciertas.

Una demostración más simple del mismo fenómeno es la que se presenta más abajo considerando jugadas de dos resultados. Este ejemplo también se basa en Allais. 
Problema 3:

A: $(4000,80)$ ó B: $(3000)$.

$\mathrm{N}=95 \quad(20)$

$(80)^{*}$

Problema 4:

$\mathrm{C}:(4000, .20)$ ó $\mathrm{D} *(3000, .25)$
$\mathrm{N}=95 \quad(65) *$

En estos dos problemas, al igual que en todos los pares de problemas presentados en esta sección, más de la mitad de los sujetos violan la teoría de la utilidad esperada. Para demostrar que el patrón modal de preferencias de los problemas 3 y 4 no es compatible con la teoría, tomemos $\mathrm{u}(0)=0$, y considerando que la elección de $\mathrm{B}$ implica $\mathrm{u}(3000) / \mathrm{u}(4000)>4 / 5$, y la elección de $C$ implica la desigualdad inversa. Fíjese que la alternativa $C=(4000,20)$ puede expresarse como (A,.25), mientras que la alternativa $\mathrm{D}=(3000,25)$ puede ser representada como (B,25). El axioma de sustitución de la teoría de utilidad sostiene que si $B$ se prefiere a $A$, entonces cualquier combinación $(B, p)$ se preferirá a la combinación ( $A, p)$. Nuestros sujetos violaron el axioma. Aparentemente, la reducción de la probabilidad de ganar de 1.0 a .25 tiene un efecto mayor que la reducción de .8 a .2 . El siguiente par de problemas ilustra el efecto de certidumbre con resultados no monetarios.

\section{Problema 5:}

A: 50 por 100 de posibilidades de ganar un viaje de tres semanas por Inglaterra, Francia e Italia.

B: Un viaje por Inglaterra durante una semana, con seguridad.

$$
\begin{array}{ll}
\mathrm{N}=72 & (22) \text { para A } \\
& (78) * \text { para B }
\end{array}
$$

Problema 6:

C: 5 por 100 de posibilidades de ganar un viaje de tres semanas por Inglaterra, Francia e Italia.

D: 10 por 100 de posibilidades de ganar un viaje de una semana por Inglaterra.

$$
\begin{array}{ll}
\mathrm{N}=72 & (67) * \text { para A } \\
& \text { (33) para B }
\end{array}
$$

El efecto de certidumbre no es el único tipo de violación del axioma de sustitución. Otra situación en que se viola el axioma se ilustra en los siguientes problemas.

Problema 7:
A: $(6000, .45)$
B: $(3000, .90)$
$\mathrm{N}=66$
(14)
$(86) *$ 
Problema 8:
C: $(6000, .001)$
$\mathrm{D}:(3000, .002)$
$\mathrm{N}=66$
$(73)^{*}$

Fíjese que en el problema 7 las probabilidades de ganar son considerablemente altas $(.90$ y.45) y la mayoría de la gente elige la alternativa donde la probabilidad de ganar es mayor. En el problema 8, existe una posibilidad de ganar, a pesar de que las probabilidades de ganar son pequeñas (.002 y .001) en las dos alternativas. En esta situación, donde existe una posibilidad de ganar que no es probable, la mayoría de la gente elige la alternativa que ofrece la mayor cantidad a ganar. Mc Crimmon y Larson encontraron resultados similares.

Los problemas anteriores ilustran las actitudes normales hacia el riesgo o la probabilidad y éstas no se pueden explicar por el modelo de la utilidad esperada. Los resultados sugieren una generalización empírica donde el axioma de sustitución es violado. Si (y,pq) es equivalente a $(\mathrm{x}, \mathrm{p})$ entonces $(\mathrm{y}, \mathrm{pq}), 0<\mathrm{p}, \mathrm{q}, \mathrm{r}<1$. Esta propiedad se incorpora a una teoría alternativa, teoría que se desarrolla en la segunda parte de este artículo.

\section{El efecto de reflexión}

La sección anterior discutió las preferencias entre las alternativas positivas, es decir, alternativas que no implicaban pérdidas. ¿Qué ocurre cuando los signos de los resultados se invierten de manera que las ganancias se convierten en pérdidas? La columna de la izquierda, de la tabla 1, muestra cuatro de los problemas de elección discutidos anteriormente, en la columna de la derecha se muestra los problemas de elección con los signos de los resultados invertidos. - $\mathrm{X}$ denota pérdidas de $\mathrm{X}, \mathrm{y}$ 《 $>$ » se utiliza para denotar la preferencia dominante, por ejemplo, la elección hecha por la mayoría de los sujetos.

TABLA 1

Preferencias entre alternativas positivas $y$ negativas

\begin{tabular}{|c|c|c|}
\hline \multicolumn{2}{|l|}{ A. Positivas } & A. Negativas \\
\hline $\begin{array}{lc}\text { Problema } & 3: \\
\mathrm{N}=95 & (4,00, .80) \\
& (20)\end{array}$ & $\begin{array}{l}(3,000) \\
(80)^{*}\end{array}$ & $\begin{array}{lcc}\text { Problema 3: } & (-4,000,80) & (-3,000) \\
=95 & (92)^{*} & (8)\end{array}$ \\
\hline $\begin{array}{l}\text { Problema 4: }(4,000,20) \\
=95 \quad(65)^{*}\end{array}$ & $\begin{array}{l}(3,000, .25) \\
(35)\end{array}$ & 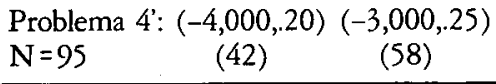 \\
\hline $\begin{array}{lc}\text { Problema } 7: & (3,000, .90) \\
N=66 & (86)^{*}\end{array}$ & $\begin{array}{l}(6,000,45) \\
(14)\end{array}$ & $\begin{array}{lcc}\text { Problema } & 7^{\prime}: \\
\mathrm{N}=66 & (-3,000, .90) & (-6,000,45) \\
& (8) & (92)^{*}\end{array}$ \\
\hline $\begin{array}{l}\text { Problema 8: } \\
\mathrm{N}=66\end{array}$ & $\begin{array}{l}(6,000, .001) \\
(73)^{*}\end{array}$ & 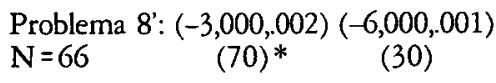 \\
\hline
\end{tabular}

En cada uno de los cuatro problemas de la tabla 1, la preferencia entre las alternativas negativas es la imagen en espejo de las preferencias entre las alternativas positivas. Así, la reflexión de las alternativas alrededor de 0 invierte el orden de preferencias. $A$ este patrón lo hemos llamado efecto de reflexión.

Volvamos ahora a las implicaciones que puedan tener nuestros datos. Primero, note que el efecto de reflexión implica que la aversión al 
riesgo en el dominio positivo, se acompaña por la atracción al riesgo en el dominio negativo. En el problema 3', por ejemplo, la mayoría de los sujetos tendían a aceptar el riesgo de .80 de perder 4000 frente a una pérdida segura de 3000 , a pesar de que la primera opción tiene - un valor esperado más pequeño. Markowitz ya señaló el hecho de aversión al riesgo en elecciones que implican alternativas negativas. Los datos de Williams mostraron que el cambio del signo de los resultados produce un cambio dramático de la aversión al riesgo a la atracción por éste. Por ejemplo, sus sujetos eran indiferentes entre las opciones $(100,65 ;-100, .35)$ y $(0)$, mostrando una aversión por el riesgo; también eran indiferentes entre $(-2000,80)$ y $(-100)$ indicando atracción por el riesgo. Una revisión reciente de Fishburn y Kochenberger muestra la dominancia de la atracción al riesgo en elecciones entre alternativas negativas.

Segundo, hay que recordar que las preferencias, en la tabla 1 , entre las alternativas positivas son inconsistentes con la teoría de la utilidad esperada. De la misma manera, las preferencias entre las alternativas negativas correspondientes también violan el principio de esperanza, por ejemplo, en los problemas 3' y 4', al igual que en los problemas 3 y 4 , se demuestra cómo los resultados que se obtienen con certeza son valorados más con respecto a los resultados inciertos. En el dominio positivo, el efecto de certidumbre contribuye a una preferencia por la aversión al riesgo para ganancias seguras sobre aquellas ganancias mayores que son solamente probables. En el dominio negativo, este mismo efecto conduce a preferencias por la atracción al riesgo para una pérdida que es solamente probable sobre pérdidas menores seguras. El mismo principio psicológico - el sobrevalorar más la certidumbre- favorece la aversión al riesgo en el dominio de las ganancias y la atracción por el riesgo en el dominio de las pérdidas.

Tercero, el efecto de reflexión descarta que la aversión por la incertidumbre o la variabilidad sea la explicación del efecto de certidumbre. Considere, por ejemplo, las preferencias prevalentes para (3000) sobre $(4000, .80)$ y para $(4000,20)$ sobre $(3000,25)$. Para resolver esta inconsistencia aparente se podría invocar al supuesto de que la agente prefiere las alternativas que tengan un valor esperado alto y una varianza pequeña (ver, por ejemplo, Allais, Markowitz, Tobin). Debido a que $(3000)$ no tiene ninguna varianza mientras que $(4000,80)$ tiene una varianza alta, la alternativa anterior podría ser elegida a pesar de su bajo valor esperado. Cuando se reducen las alternativas, la diferencia de varianza entre $(3000,25)$ y $(4000,20)$ puede ser insuficiente para superar la diferencia en valor esperado. Debido a que $(-3000)$ tiene tanto un valor esperado mayor como una varianza menor que $(-4000, .80)$, se supone que se preferirá la pérdida segura, contrariamente a lo que muestran los datos. Así, nuestros datos son incompatibles con la noción de que la certeza se prefiere siempre. Más bien parece que la certeza aumenta la aversión por las pérdidas y la atracción por las ganancias.

\section{Seguro probabilístico}

La adquisición de un seguro para evitar pequeñas o grandes pérdidas se ha considerado una fuerte evidencia a favor de la concavidad de la función de utilidad para el dinero. Sin embargo, ¿por qué, a pesar de todo, la gente gasta tal cantidad de dinero en la compra de pólizas 
de seguros a un precio que excede al coste esperado? Sin embargo, un examen detallado de la atracción de las distintas formas de seguro no apoya la noción de que la función de utilidad para el dinero, sea siempre cóncava. Por ejemplo, la gente a menudo prefiere programas de seguro que ofrezcan una cobertura limitada con una deducción baja o nula si se compara con aquellas pólizas que ofrecen una máxima cobertura, con deducciones altas -contrariamente a la aversión por el riesgo (ver, por ejemplo, Fuchs). Otro tipo de problema, con respecto a los seguros donde las respuestas de las personas son inconsistentes con la hipótesis de concavidad, la podemos denominar como seguro probabilístico. Para ilustrar este concepto, considere el siguiente problema presentado a 95 estudiantes de la Universidad de Stanford.

Problema 9: Suponga que tiene que considerar la posibilidad de asegurar una propiedad por accidente, por ejemplo, fuego o robo. Después de examinar los riesgos y el precio de la póliza se da cuenta de que no tiene ninguna preferencia clara entre las opciones de comprar el seguro o dejar la propiedad sin asegurar.

En ese momento llama su atención el hecho de que la compañía de seguros ofrece un nuevo programa llamado seguro probabilístico. En este programa se paga la mitad del precio normal. En caso de accidente, existe un 50 por 100 de posibilidades de que pague la otra midad del precio y de que la compañía le cubra todas las pérdidas, y otro 50 por 100 de posibilidades de que se le devuelva el importe del seguro y de que sufra todas las pérdidas. Por ejemplo, si ocurre un accidente en un día par del mes se paga la otra mitad del importe y se cubren los daños, pero si el accidente ocurre en un día impar del mes, el importe del seguro le será devuelto y no se cubren las pérdidas.

Recuerde que el importe para que exista una cobertura total es tal que duda en suscribir la póliza.

Bajo estas circunstancias, ¿compraría el seguro probabilístico?

$\begin{array}{ccc} & \text { si } & \text { no } \\ N=95 & (20) & (80)^{*}\end{array}$

A pesar de que el problema 9 puede parecer rebuscado, merece la pena destacar que el seguro probabilístico representa varias formas de una acción protectora cuando uno paga un cierto precio para reducir la probabilidad de que ocurra un hecho no deseable (sin eliminarlo del todo). Tanto la instalación de una alarma contra atracos como la sustitución de las ruedas viejas y la decisión de dejar de fumar, son ejemplos que pueden ser analizados como un seguro probabilístico.

Los sujetos que respondieron al problema 9 y a las distintas variantes de la misma pregunta indican que, generalmente, no es atractivo el seguro probabilístico. Aparentemente la reducción de la probabilidad de una pérdida de $\mathrm{p}$ a $\mathrm{p} / 2$ se valora menos que la reducción de la probabilidad de esa pérdida de $\mathrm{p} / 2$ a 0 .

En contraste con los datos, la teoría de la utilidad esperada (con una $U$ cóncava) implica que el seguro probabilístico es superior al seguro normal. Es decir, si la situación económica w es tal que se prefiere pagar una póliza $\mathrm{Y}$ para asegurarse de una pérdida $\mathrm{X}$ con una probabilidad $p$, entonces definitivamente se preferirá pagar un coste menor ry para reducir la probabilidad de una pérdida $x$ de $p$ a $(1-r) p$, $0<\mathrm{r}<1$. Formalmente si se es indiferente entre $(w-x, p ; w, 1-p)$ y $(w-y)$, entonces se debería elegir el seguro probabilístico $(w-x,(1-r) p ; w-y, r p ; w-r y, 1-p)$ sobre el seguro normal $(w-y)$. 
Para probar esta proposición, demostramos que

$$
\mathrm{pu}(\mathrm{w}-\mathrm{x})+(\mathbf{l}-\mathrm{p}) \mathrm{u}(\mathrm{w})=\mathrm{u}(\mathrm{w}-\mathrm{y})
$$

implica

$$
(1-r) p u(w-x)=r p u(w-y)+(1-p) u(w-r y) \quad>u(w-y) .
$$

Sin pérdida de generabilidad podemos establecer $u(w-x)=0 y$ $u(w)=1$. Así, $u(w-y)=1-p$, y queremos demostrar que

$$
\begin{aligned}
& \mathrm{rp}(1-\mathrm{p})+(1-\mathrm{p}) \mathrm{u}(\mathrm{w}-\mathrm{ry})>1-\mathrm{p} \text { ó } \\
& \mathrm{u}(\mathrm{w}-\mathrm{ry})>1-\mathrm{rp}
\end{aligned}
$$

que se mantiene si, y sólo si, u es cóncava.

Esta consecuencia es bastante desconcertante con respecto a la hipótesis de la aversión por el riesgo de la teoría de la utilidad, ya que el seguro probabilístico parece ser intuitivamente más arriesgado que un seguro normal en el cual se elimina enteramente el elemento de riesgo. Evidentemente, la noción intuitiva de riesgo no está especificada de manera adecuada por la concavidad de la función de utilidad para el dinero.

La aversión del seguro probabilístico es particularmente complicada ya que todo seguro es, en esencia, probabilístico. El más ferviente comprador de seguros será vulnerable a muchos riesgos financieros y de otro tipo que sus pólizas no cubran. Parecen existir diferencias significativas entre el seguro probabilístico y lo que se conoce como seguro contingente que posibilita la seguridad de la cobertura para riesgos de tipos específicos; compare, por ejemplo, el seguro probabilístico frente a todas las formas de pérdida o daño de sus inmuebles y un seguro contingente que elimine todos los riesgos de pérdida por robo, por ejemplo, pero que no cubre otros riesgos, vg., por fuego. Supongamos que el riesgo contingente será generalmente más atractivo que el seguro probabilístico cuando las probabilidades de pérdidas no protegidas sean equiparables. Así, dos alternativas que son equivalentes en probabilidad y resultados podrán tener valores diferentes dependiendo de nuestra formulación. En la siguiente sección describimos diversas demostraciones de este fenómeno general.

\section{Efecto de aislamiento}

Con el fin de simplificar la elección entre alternativas, la gente normalmente descuida los componentes que las alternativas comparten, y atienden a los componentes que las distinguen (Tversky) (44)). Esta aproximación a los problemas de elección puede producir preferencias inconsistentes debido a que un par de alternativas puede descomponerse en componentes comunes y distintivos de más de una manera, y descomposiciones diferentes, en ocasiones, conducen a preferencias diferentes. Nos referimos a este fenómeno con el nombre de efecto de aislamiento.

Problema 10: Considera las siguientes fases de estas dos jugadas. En la primera fase existe una probabilidad de .75 de terminar la jugada sin haber ganado nada, y una probabilidad de .25 de pasar a la segunda fase. Si se llega a esta segunda fase se tendrá que elegir entre 
La elección debe ser hecha antes de empezar a jugar, por ejemplo, antes de que el resultado de la primera fase se conozca.

Fíjese que en esta jugada se tiene que elegir entre $.25 \times .80=.20$ de probabilidad de ganar 4000 , y $.25 \times 1.0=.25$ de probabilidad de ganar 3000. Así, en términos de resultados finales y de probabilidades se enfrenta a una elección entre $(4000,20)$ y $(3000,25)$, al igual que en el problema 4. De cualquier manera, las preferencias dominantes son diferentes en estos dos problemas. De los 141 sujetos que respondieron al problema 10 , el 78 por 100 eligió la última alternativa, al contrario que la preferencia modal del problema 4. Evidentemente, la gente ignora la primera fase de la jugada cuyos resultados son compartidos por las dos alternativas, y consideran el problema 10 como una elección entre $(3000)$ y $(4000,80)$ como en el anterior problema 3 .

Las formulaciones corrientes y secuenciales del problema 4 se presentan en un árbol de decisión en la figura 1 y 2 , respectivamente. Siguiendo la convención usual, los cuadrados denotan los nodos de decisión y los círculos el cambio de nodos. La diferencia esencial entre las dos representaciones radica en el lugar donde se encuentran los nodos de decisión. En la forma corriente (figura 1), el decisor se enfrenta a una elección entre dos alternativas arriesgadas, mientras que en la forma secuencial (figura 2) se enfrenta a una elección entre una alternativa arriesgada y otra sin riesgo. Esta forma secuencial se realiza introduciendo una dependencia entre las alternativas, sin cambiar ni sus probabilidades ni sus resultados.

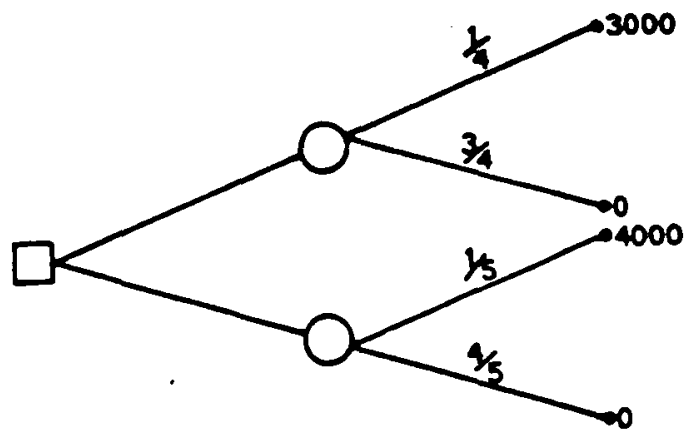

Figura 1.

Representación del problema 4 en un árbol de decisión (formulación corriente).

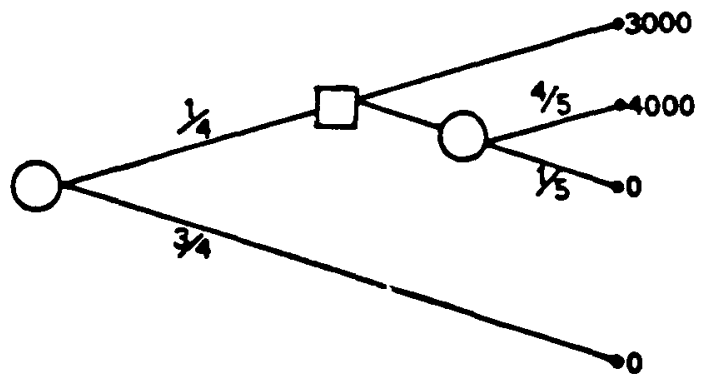

FIGURA 2.

Representación del problema 10 en un árbol de decisión (formulación secuencial). 
eventos son independientes en la formulación corriente. De esta manera, el resultado de ganar 3000 tiene una clara ventaja en la formulación secuencial que no tiene en la formulación corriente.

Particularmente significativa es la inversión de preferencias debida a la dependencia entre sucesos, ya que se viola la suposición básica del análisis teórico de la decisión; es decir, el que las elecciones entre alternativas estén únicamente determinadas por las probabilidades de los estados finales.

Es fácil pensar en problemas de decisión que estén más representados en una de las formas anteriores que en la otra. Por ejemplo, la elección entre dos especulaciones arriesgadas diferentes puede analizarse mejor en la forma corriente. Por otro lado, el siguiente problema se asemeja más a una representación en la forma secuencial. Se puede invertir dinero en una empresa, en caso de que la empresa quiebre se pierde el capital invertido, y en el caso contrario se tiene una opción entre que te devuelvan una cantidad de dinero fija o un porcentaje sobre los beneficios adquiridos. El efecto de aislamiento conlleva que se prefiera una empresa en la que una de sus opciones consiste en la recuperación segura de una cantidad de dinero fija respecto de otra donde en ninguna de sus opciones se incluye una recuperación de una cantidad fija.

El problema anterior muestra cómo las preferencias pueden cambiarse por distintas representaciones de probabilidades. A continuación demostramos que las elecciones pueden alterarse debido a variaciones en la representación de los resultados.

Considere los siguientes problemas, presentados a dos grupos distintos de sujetos.

Problema 11: Además de lo que posea se le van a dar 1000. Elija, ahora, entre

$$
\begin{array}{ll}
A:(1000,5) \text { y } & \text { B: }(500) \\
N=70 \quad(16) & (84)^{*}
\end{array}
$$

Problema 12: Además de lo que posea se le van a dar 2000. Elija entre

$$
\begin{array}{ll}
C:(-1000, .50) \text { y } & D:(-500) \\
N=68 & (69)^{*}
\end{array}
$$

La mayoría de los sujetos eligieron $\mathrm{B}$ en el primer problema y $\mathrm{C}$ en el segundo. Estas preferencias confirman el efecto de reflexión que se había observado en la tabla 1 en la que se muestra la aversión al riesgo para alternativas positivas y la atracción al riesgo en las negativas. De cualquier manera, fíjese que cuando se analiza en términos de los estados finales, los dos problemas de elección son idénticos. Concretamente,

$$
A=(2000, .50 ; 1000, .50)=C
$$

y

$$
B=(1500)=D .
$$

De hecho, el problema 12 se harobtenido del problema 11 al añadirle 1000 al bono inicial y sustrayendo 1000 de todos los resultados. 
Evidentemente, los sujetos no integraron el bono inicial con las alternativas presentadas. El bono no se incluyó en la comparación de las alternativas ya que era común para las dos opciones de cada uno de los problemas.

El patrón de los resultados de los problemas 11 y 12 es claramente inconsistente con la teoría de la utilidad. En esta teoría, por ejemplo, se asigna la misma utilidad a la cantidad de $1000000 \$$, sin considerar que el estado inicial del sujeto pudiera ser $95000 \$$ o $105000 \$$. En consecuencia, la elección entre una cantidad total de 100000 y la posibilidad de ganar $95000 \$$ y $105000 \$$ debería ser independiente de si se tiene una cantidad mayor o menor a las anteriormente mencionadas. Con la introducción del supuesto de aversión por el riesgo, la teoría subraya que la certeza de ganar $100000 \$$ se deberá preferir siempre antes que la jugada. Sin embargo, los sujetos del problema 12, así como otras respuestas previas, sugieren que este patrón se obtendrá si los sujetos poseen cantidades más pequeñas, pero no si poseen cantidades más grandes.

La desatención aparente hacia el bono incial que se observa tanto en el problema 11 como en el 12, parece indicar que el valor o utilidad se deben más a cambios de la cantidad monetaria que a las posiciones finales en las que se incluye la cantidad que se poseía. Esta conclusión es la piedra angular de una teoría alternativa de la elección bajo riesgo, la cual se describe en las secciones siguientes.

\section{TEORIA}

Las secciones anteriores han revisado distintos efectos empíricos que invalidaban la teoría de la utilidad esperada como modelo descriptivo. A partir de aquí vamos a presentar un modelo alternativo para explicar la toma de decisiones individual bajo riesgo, modelo que hemos llamado teoría prospectiva. Esta teoría se ha desarrollado para alternativas simples con resultados monetarios y probabilidades dadas; sin embargo puede ampliarse a otras elecciones. La teoría prospectiva distingue dos fases en el proceso de elección: una primera fase de preparación y una fase subsecuente de evaluación. En la fase de preparación se realiza un análisis preliminar de las alternativas que se presentan, análisis que, generalmente, consiste en una representación múltiple de las alternativas. En la segunda fase, las alternativas anteriores son evaluadas y se eligirá la alternativa con mayor valor. A continuación, describiremos la fase de preparación, y desarrollaremos un modelo formal de la fase de evaluación.

La función de la fase de preparación es la de organizar y reformular las opciones de tal manera que se simplifique posteriormente la evaluación y la elección. La preparación consiste en la aplicación de diversas operaciones que transforman los resultados y las probabilidades asociadas con las alternativas que se presentan. Las operaciones más importantes de esta fase se describen a continuación.

Codificación. Como se ha mostrado en la sección anterior la gente, normalmente, percibe los resultados bien como ganancias o bien como pérdidas, más que un estado final de su bienestar o estado de riqueza. Por supuesto, las ganancias y las pérdidas se definen de acuerdo a un punto de referencia neutral. Este punto de referencia generalmente corresponde con la posición que se tiene en ese momento, en este caso las ganancias y las pérdidas coinciden con la cantidad que en ese mo- 
mento se recibe o se paga. De cualquier manera, la localización del punto de referencia, y la subsiguiente codificación de los resultados como ganancias o pérdidas, puede verse afectada por la formulación de las alternativas que se ofrecen y por las expectativas del decisor.

Combinación. Las alternativas, a menudo, pueden simplificarse combinando las probabilidades asociadas con resultados idénticos. Por ejemplo, la alternativa $(200,25 ; 200,25)$ se reducirá a $(200,50)$ y se evaluará de esta manera.

Separación. Algunas alternativas contienen componentes que no implican riesgo que son separados de los componentes con riesgo en la fase de preparación. Por ejemplo, la alternativa $(300, .80 ; 200,20)$ se descompone naturalmente en una ganancia segura de 200 y en una alternativa arriesgada $(100,80)$. De la misma manera, la alternativa $(-400, .40 ;-100,60)$ se interpreta como una pérdida segura de $100 \mathrm{y}$ una alternativa de $(-300, .40)$.

Las operaciones anteriores se aplican a cada una de las alternativas separadamente. La siguiente operación se aplica a un conjunto de dos o más alternativas.

Cancelación. Lo fundamental del efecto de aislamiento, descrito anteriormente, es la eliminación de los componentes que son compartidos por las alternativas que se ofrecen. Así, nuestros sujetos ignoraban, aparentemente, la primera fase del juego secuencial que se presentaba en el problema 10; ya que esta fase era común a las dos opciones, y se evaluaban las alternativas con respecto a los resultados de la segunda fase (ver figura 2). De manera similar, se ignoró el bono común que se añadió a las alternativas en los problemas 11 y 12 . Otro tipo de cancelación implica la eliminación de los elementos comunes, verbigracia, pares de resultados-probabilidades. Por ejemplo, la elección entre $(200, .20 ; 100, .50 ;-50, .30)$ y $(200, .20 ; 150, .50 ;-100, .30)$ puede reducirse por la cancelación de una elección entre $(100, .50 ;-50, .30)$ y $(150, .50 ;-100,30)$.

Existen otras dos operaciones que merecen ser mencionadas: la simplificación y la detección de la dominancia. La primera de ellas se refiere a la simplificación de las alternativas redondeando las probabilidades o los resultados. Por ejemplo, la alternativa $(101, .49)$ es considerada como un 50 por 100 de posibilidades de ganar 100 . Una forma especial de simplificación es aquella que elimina los resultados que son extremadamente poco probables. La segunda operación implica la exploración de las alternativas que se ofrecen para detectar las alternativas dominadas, las cuales se rechazan sin la evaluación posterior.

Debido a que las operaciones de preparación facilitan la tarea de decisión, se supone que se utilizarán siempre que sea posible. De cualquier manera, algunas operaciones de la fase de preparación pueden permitir o impedir la aplicación de otras. Por ejemplo, (500,.20; $101, .49)$ parecerá ser dominante a $(500,15 ; 99,51)$ si la segunda parte de ambas alternativas se simplifica a $(100,50)$. La alternativa final podrá depender de la secuencia de las operaciones de preparación, que generalmente varían con la estructura de los conjuntos que se ofrecen y con el formato en el que se presentan. Un estudio detallado de este problema está fuera de los límites de este trabajo. En este artículo se discuten los problemas de elección donde aparece razonable asumir que: o la formulación original de las alternativas no permite preparaciones posteriores, o que las alternativas preparadas pueden especificarse sin ambigüedad. 
Muchas de las anomaliáas acerca de las preferencias se deben a la preparación de las alternativas. Por ejemplo, las inconsistencias asociadas con el efecto de aislamiento resultan de la cancelación de los componentes comunes. Algunas intransitividades de la elección son explicadas por una simplificación que se produce al eliminar las pequeñas diferencias existentes entre alternativas (ver Tversky). Generalmente, el orden de preferencia entre las alternativas no necesita ser invariante a través del contexto, debido a que la alternativa que se ofrece puede ser preparada de distintas maneras dependiendo del contexto en el cual se presenta.

A partir de la fase de preparación, se supone que el decisor evaluará cada una de las alternativas "preparadas» anteriormente, y elegirá la alternativa que presente el valor mayor. El valor total de la alternativa preparada, denotada por $\mathrm{V}$, se expresa en términos de dos escalas, $\pi$ y v.

La primera escala, $\pi$, asocia con cada probabilidad $\mathrm{p}$ un peso de decisión $\pi(\mathrm{p})$, que refleja el impacto de p sobre el valor total de la alternativa. De cualquier manera, $\pi$ no es una medida de probabilidad, $y$, posteriormente, se demostrará que $\pi(p)+\pi(1-p)$ es menor que la unidad. La segunda escala, $\mathrm{V}$, asigna a cada resultado $\mathrm{x}$ un número $\mathrm{v}(\mathrm{x})$, que refleja el valor subjetivo del resultado. Recuérdese que los resultados se definen en relación con el punto de referencia, el cual sirve de punto cero del valor de la escala. De esta manera, $\mathrm{v}$ mide los valores de las desviaciones desde el punto de referencia, es decir, pérdidas y ganancias.

La presente formulación se refiere a alternativas simples de la forma (x,p;y,q), las cuales tienen, por lo menos, dos resultados que no son cero. En estas alternativas, se recibe $x$ con una probabilidad de $p$, y con una probabilidad de $\mathrm{q}$, y nada con una probabilidad $1-\mathrm{p}-\mathrm{q}$, donde $p+q<=1$. Una alternativa es estrictamente positiva si sus resultados son todos positivos, por ej., si $\mathrm{x}, \mathrm{y}>0$ y $\mathrm{p}+\mathrm{q}=1$; es estrictamente negativa si sus resultados son todos negativos. Una alternativa será regular si no es ni estrictamente positiva ni estrictamente negativa.

La ecuación básica de la teoría describe la manera en la que $\pi$ y $\mathrm{v}$ son combinados para determinar el valor total de las alternativas regulares.

Si $(x, p ; y, q)$ es una alternativa regular (por ej., tanto si $p^{+} q<1$, o $\mathrm{x}>=0>=\mathrm{y}$, ó $\mathrm{x}<=0<\mathrm{y}$ ), entonces

$$
\mathrm{V}(\mathrm{x}, \mathrm{p} ; \mathrm{y}, \mathrm{q})=\pi(\mathrm{p}) \mathrm{v}(\mathrm{x})+\pi(\mathrm{q}) \mathrm{v}(\mathrm{y})
$$

donde $v(0)=0, \pi(0)=0, y \boldsymbol{\pi}(1)=1$. Como en la teoría de la utilidad, $\mathrm{V}$ se define sobre las alternativas, mientras que $\mathrm{v}$ se define sobre los resultados. Las dos escalas coinciden para las alternativas seguras, donde $\mathrm{V}(\mathrm{x}, 1.0)=\mathrm{V}(\mathrm{x})=\mathrm{v}(\mathrm{x})$.

La ecuación (1) generaliza la teoría de la utilidad esperada relajando el principio de esperanza. Un análisis axiomático de esta representación se muestra en el apéndice, donde se describen las condiciones que aseguran la existencia de un único $\pi$ y una escala de razón $v$ que satisface la ecuación (1).

La evaluación de las alternativas estrictamente positivas y de las que son estrictamente negativas sigue reglas diferentes. En la fase de preparación estas alternativas se separan en dos componentes: (i) el componente sin riesgo, por ej., la ganancia mínima o la pérdida segura 
que se obtendrá o pagará; (ii) el componente arriesgado, por ej., la ganancia adicional o la pérdida que está realmente en juego. La evaluación de tales alternativas se describe en la ecuación siguiente:

Si $p+q=1$ y si $x>y>0$ o $x<y<0$, entonces

$$
V(x, p ; y, q)=v(y)+\pi(p)(v(x)-v(y))
$$

Es decir, el valor de una alternativa estrictamente positiva o estrictamente negativa es igual al valor del componente sin riesgo más el valor de la diferencia entre los resultados, multiplicado por el peso asociado con el resultado más extremo. Por ejemplo, $\mathrm{V}(400, .25 ; 100, .75)=$ $\mathrm{v}(100)+\pi(.25)(\mathrm{v}(400)-\mathrm{v}(100))$. La característica principal de la ecuación (2) radica en que el peso de decisión se aplica al valor de la diferencia $v(x)-v(y)$, que representa el componente arriesgado de la alternativa, pero no a $v(y)$, que representa el componente sin riesgo, nótese que la parte derecha de la ecuación (2) es igual a $\pi(\mathrm{p}) \mathrm{v}(\mathrm{x})+(1-\pi(\mathrm{p}) \mathrm{v}(\mathrm{y})$. Así, la ecuación (2) queda reducida a la ecuación $(1)$ si $\pi(\mathrm{p})+\pi(1-\mathrm{p})=1$. Como veremos más tarde, esta condición generalmente no se satisface.

En intentos anteriores de modificación de la teoría de la utilidad esperada ya habían aparecido muchos de los elementos del modelo de evaluación de Markowitz, que fue el primero en proponer que la utilidad se definiría sobre las ganancias y las pérdidas más que por las posiciones finales, un supuesto que ha sido implícitamente aceptado en la mayoría de las medidas empíricas de la utilidad. También, Markowitz señaló la presencia de la atracción por el riesgo en preferencias entre alternativas tanto positivas como negativas, y propuso una función de utilidad con regiones convexas y cóncavas tanto en los dominios positivos como en los negativos. Su tratamiento, de cualquier manera, mantiene el principio de esperanza; por esto no puede explicar muchas de las violaciones de este principio (ver, por ejemplo, tabla 1).

La sustitución de las probabilidades por pesos más generales fue propuesta por Edwards, y este modelo ha sido investigado en diversos estudios empíricos. Modelos parecidos se han desarrollado por Fellner, que introdujo el concepto de peso de decisión para explicar la aversión a las ambigüedades; y por Van Dam, que intentó escalar los pesos de decisión. Otros modelos alternativos de elección que analizan críticamente la teoría de la utilidad esperada, son los de Allais, Coombs, Fishburn y Hansson.

Las ecuaciones de la teoría prospectiva mantienen la forma bilineal general de la teoría de la utilidad esperada. De todas maneras, con el fin de acomodar los efectos descritos en la primera parte de este artículo, asumimos que los valores están ligados más a los cambios que a los estados finales, y que los pesos de decisión no coinciden con las probabilidades dadas. Estas diferencias con la teoría de la utilidad espera$\mathrm{da}$, conducen a consecuencias normativamente inaceptables, como inconsistencias, intransitividades y violaciones de la dominancia. Estas anomalías de la preferencia son generalmente corregidas por el decisor al darse cuenta que sus preferencias son inconsistentes, intransitivas o inadmisibles. Pero en muchas situaciones, el decisor no tiene la oportunidad de descubrir que sus preferencias podrian violar algunas reglas de decisión que le gustaría seguir. En estas circunstancias se espera que ocurran las anomalías que implica la teoría prospectiva. 
Una de las características de la presente teoría radica en asumir que los valores son cambios en la riqueza o en el bienestar más que en los estados finales. Este supuesto es compatible con los principios básicos acerca de juicio y de la percepción. Nuestro aparato perceptual se atenúa en la evaluación de los cambios o diferencias más que por la evaluación de magnitudes absolutas. Al responder a atributos tales como el brillo, el volumen, o la temperatura, el contexto presente y pasado de la experiencia define un nivel de adaptación, o un punto de referencia, y los estímulos se perciben en relación con el punto de referencia. Así, un objeto con una temperatura dada puede percibirse como caliente o como frío al tacto dependiendo de la temperatura a la que se está adaptado. El mismo principio se aplica a atributos no-sensoriales tales como la salud, el prestigio y la riqueza. Por ejemplo, el mismo nivel de riqueza puede implicar una miseria extrema para una persona y una gran riqueza para otra, dependiendo de su haberes actuales. El énfasis que se da a los cambios como portadores del valor no implica que el valor de un determinado cambio sea independiente de la posición inicial. Estrictamente, el valor debe ser tratado como una funnción de dos argumentos: la posición de medida que sirve como punto de referencia, y la magnitud del cambio (positivo o negativo) con respecto al punto de referencia. Por ejemplo, una actitud individual hacia el dinero puede describirse como un libro, donde cada página presenta la función de valoración para los cambios respecto a una determinada posición. Claramente, las funciones de valoración descritas en páginas diferentes no son idénticas: probablemente se hacen más lineales a medida que aumenta el valor de la posición de medida. No obstante, el orden de preferencia de las alternativas no sufre grandes variaciones debidas a cambios pequeños o incluso moderados en la posición de medida. La seguridad equivalente de la alternativa $(1000, .50)$, por ejemplo, descansa entre 300 y 400 para la mayoría de la gente, en un rango amplio de las posiciones de medida. Consecuentemente, la representación del valor en función de un solo argumento, por lo general, proporciona una aproximación satisfactoria.

La propiedad de que la respuesta psicológica es una función cóncava de la magnitud de los cambios psicológicos es compartida por muchas dimensiones sensoriales y perceptuales. Por ejemplo, es más fácil discriminar entre un cambio de temperatura en una habitación de 3 a 6 grados que discriminar entre un cambio de 13 a 16 grados. Proponemos que este principio se aplica de forma particular a la evaluación de cambios monetarios. Así, la diferencia en valor entre una ganancia de 100 y una ganancia de 200 parece ser mayor que la diferencia entre una ganancia de 1100 y otra de 1200 . De forma parecida, la diferencia entre una pérdida de 100 y una pérdida de 200 parece mayor que la diferencia de una pérdida entre 1100 y 1200 , a no ser, que la pérdida más grande sea intolerable. De esta manera, hipotetizamos que la función de valoración para cambios de riqueza es normalmente cóncava, por encima del punto de referencia $(v "(x)<0$, para $x>0)$; y generalmente convexa, por debajo de este punto $\left(v^{\prime \prime}(x)>0\right.$, para $\left.x<0\right)$. Es decir, el valor marginal de pérdidas y ganancias generalmente decrece con su magnitud. Galanter y Pilner (17) apoyàron esta hipótesis, estos autores escalaron la magnitud percibida de ganancias y pérdidas tanto monetarias como no monetarias. 
La hipótesis anterior respecto a la forma de la función de valoración se ha basado en las respuestas de los sujetos hacia las pérdidas y las ganancias en un contexto arriesgado. Proponemos que la función de valor que se deriva de elecciones arriesgadas comparte las mismas características, como se muestra en los siguientes problemas.

Problema 13:

$$
\begin{array}{ll}
(6000,25) \circ & (4000,25 ; 2000, .25) \\
\mathrm{N}=68 \quad(18) & (82)^{*}
\end{array}
$$

Problema 13':

$$
\begin{array}{ll}
(-6000, .25) \text { o } & (-4000, .25 ;-2000, .25) \\
\mathrm{N}=64 & (70)^{*}
\end{array}
$$

Aplicando la ecuación 1 a la preferencia modal en estos problemas, se mantiene que

$$
\begin{aligned}
& \pi(.25) \mathrm{v}(6000)<\pi(.25)(\mathrm{v}(4000)+\mathrm{v}(2000)) \mathrm{y} \\
& \pi(.25) \mathrm{v}(-6000)>\pi(.25)(\mathrm{v}(-4000) \mathrm{v}(-2000))
\end{aligned}
$$

Por tanto, $\mathrm{v}(6000)<\mathrm{v}(4000)+\mathrm{v}(2000)$ y $\mathrm{v}(-6000)>\mathrm{v}(-4000)+$ $\mathrm{v}(-2000)$.

Estas preferencias están en consonancia con la hipótesis de que la función de valoración es cóncava para las ganancias y convexa para las pérdidas.

Cualquier discusión de la función de utilidad para el dinero debe dejar sitio para el efecto de circunstancias especiales sobre las preferencias. Por ejemplo, la función de utilidad de un sujeto que necesita $60000 \$$ para comprarse una casa deberá mostrar un excepcional incremento acelerado cerca del valor criterio. De manera similar, la aversión de un sujeto a perder puede aumentar bruscamente cerca de la pérdida que le supondría vender su casa y cambiarse a un barrio menos deseable. Así, la función de valoración (utilidad) derivada de un sujeto no siempre refleja actitudes "puras» hacia el dinero, ya que puede verse afectado por consecuencias adicionales asociadas con cantidades específicas. Estas perturbaciones pueden producir fácilmente regiones convexas para ganancias y regiones cóncavas para pérdidas en la función de valoración. El último caso parece ser más frecuente, puesto que generalmente las pérdidas de grandes cantidades producen un cambio del estilo de vida.

El hecho de que las pérdidas aparezcan como mayores que las ganancias es una característica destacada de las actitudes de los sujetos hacia el cambio de los estados de riqueza. La desesperación que puede producir la pérdida de una suma considerable de dinero nos parece mayor que la satisfacción que podríamos obtener al ganar la misma cantidad. Es más, la mayoría de la gente encuentra que apuestas simétricas de la forma (X, .50; $-\mathrm{X}, .50)$ tienen distinto atractivo. Aun más, la aversión hacia apuestas simétricas generalmente aumenta con el tamaño de la jugada. Así, si $x>y>=0$, entonces $(y, .50 ;-y, .50)$ se prefiere a $(\mathrm{x}, .50 ;-\mathrm{x}, .50)$. Según la ecuación 1

$$
\begin{aligned}
& v(y)+(-y)>v(x)+v(-x) y \\
& v(-y)-v(-x)>v(x)-v(y)
\end{aligned}
$$


Haciendo $\mathrm{y}=0$ se mantiene que $\mathrm{v}(\mathrm{x})<-\mathrm{v}(-\mathrm{x})$, y a medida que se aproxima a $x$ se mantiene que $v^{\prime}(x)<v^{\prime}(-x)$, una vez que se ha probado que existe $v$ '.

Resumiendo, proponemos que la función de valoración está (i) definida sobre las desviaciones respecto al punto de referencia (ii); es generalmente cóncava para las ganancias y convexa para las pérdidas (iii), más acelerada para las pérdidas que para las ganancias. En la figura 3 se muestra una función de valoración que satisface estas tres propiedades. Se propone una función de valoración con una forma-S, que es más acelerada en el punto de referencia a diferencia de la función de utilidad propuesta por Markowitz, que es relativamente aplanada en esta región.

FIGURA 3.

Una función de valoración bipotética.

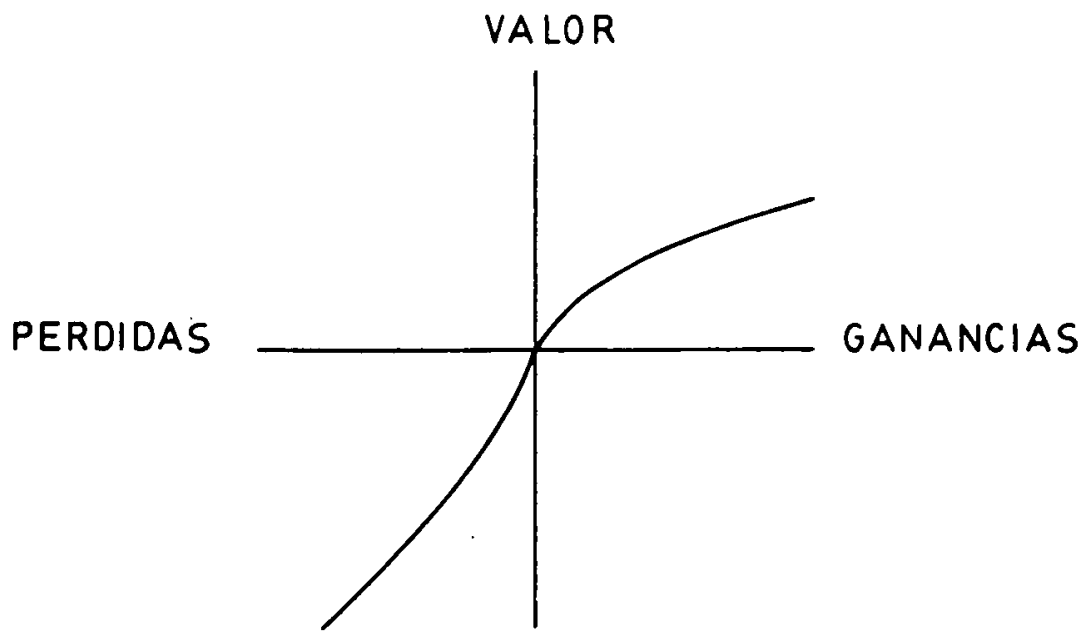

A pesar de que nuestra teoría se puede aplicar para derivar la función de valoración a partir de las preferencias entre alternativas, la forma de escalamiento actual es considerablemente más complicada que en la teoría de la utilidad debido a la introducción de los pesos de decisión. Por ejemplo, los pesos de decisión pueden producir aversión al riesgo y atracción a éste incluso con una función de valoración lineal. No obstante, es interesante señalar que se han observado las principales propiedades de la función de valoración en un análisis detallado de las funciones de utilidad de Von Neumann-Morgenstein (Fishburn y Kochenberger) para los cambios de riqueza. Las funciones se obtuvieron a partir de 30 decisores en diversos campos de negocios, en cinco estudios independientes. La mayoría de las funciones de utilidad fueron cóncavas para las ganancias y convexas para las pérdidas, y sólo tres de los sujetos manifestaron una aversión al riesgo tanto para las ganancias como para las pérdidas. Todas las funciones, excepto una, se consideraron más aceleradas para las pérdidas que para las ganancias.

\section{La función de ponderación}

En la teoría prospectiva, el valor de cada uno de los resultados se multiplica por un peso de decisión. Estos pesos se infieren a partir de las elecciones entre las alternativas de la misma manera que las pro- 
babilidades subjetivas se infieren a partir de las preferencias, en el enfoque de Ramsey-Savage. De todas formas, los pesos no son iguales a las probabilidades: no obedecen los axiomas de la probabilidad y no deben ser interpretados como medida del grado o creencia*.

Considere el siguiente juego, en el que uno puede ganar la cantidad de 1000 o nada dependiendo del lanzamiento de una moneda. Para cualquier persona sensata, la probabilidad de ganar en esta situación es de.50. Lo anterior se puede verificar de muy diversas formas, por ejemplo, demostrando que el sujeto es indiferente entre la apuesta de caras o cruces, o mediante su informe verbal que muestra que considera los dos eventos como equiprobables. Como demostraremos posteriormente, el peso de decisión $\pi(.50)$ derivado de las elecciones es probablemente menor que .50. Los pesos de decisión miden el impacto de los eventos sobre la deseabilidad de las alternativas y no simplemente la probabilidad que se percibe. Las dos escalas coinciden (por ej., $\pi(\mathrm{p})=\mathrm{p}$ ) si se mantiene el principio de esperanza, y sólo en este casor

Los-problemas de decisión que se discuten en este artículo se formularon con probabilidades numéricas explícitas, y nuestro análisis supone que los sujetos adoptaron los valores dados de p. Puesto que los eventos sólo fueron identificados por las probabilidades dadas, es posible, en este contexto, expresar los pesos de decisión como una función de la probabilidad dada. De todas formas, y, en general, los pesos de decisión relacionados con un evento podrían estar influenciados por otros factores como, por ejemplo, la ambigüedad.

Volvamos de nuevo a discutir las propiedades más destacadas de la función de ponderación, función que relaciona los pesos de decisión con las probabilidades dadas. Naturalmente, $\pi$ es una función creciente de $\mathrm{p}$, con $\pi(0)=0$ y $\pi(1)=1$. Así, se ignoran los resultados contingentes con un suceso imposible, y la escala se normaliza de tal modo que $\pi(\mathrm{p})$ es la razón del peso asociado con la probabilidad p por el peso asociado con un suceso seguro.

En primer lugar, trataremos algunas de las propiedades de la función de ponderación para probabilidades pequeñas. Las preferencias en el problema 8 y 8 ' sugieren que para valores pequeños de $p, \pi$ es una función subaditiva de $\mathrm{p}$, por ej. $\boldsymbol{\pi}(\mathrm{rp})>\mathrm{r} \pi(\mathrm{p})$ para $0<\mathrm{r}<1$. Recuerde que en el problema $8,(6000, .001)$ se prefería a $(3000, .002)$, así

$$
[\pi(.001)] /[\pi(.002)]>[\mathrm{v}(3000)] /[\mathrm{v}(6000)]>1 / 2
$$

Por la concavidad de $\mathbf{v}$

La misma conclusión se deriva de las preferencias reflejadas en el problema $8^{\circ}$. El patrón de preferencias en el problema 7 y $7^{\circ}$ sugiere, sin embargo, que la subaditividad no necesita mantenerse para los valores grandes de $\mathrm{p}$.

Es más, proponemos que las probabilidades pequeñas generalmente se ponderan más, así, $\boldsymbol{\pi}(\mathrm{p})>\mathrm{p}$ para $\mathrm{p}$ pequeño. Considere los siguientes problemas de decisión.

Problema 14:

$(5000,001) \circ(5)$

$\mathrm{N}=72 \quad(72)^{*}$ 
Fíjese que en el problema 14, la gente normalmente prefiere lo que es en sí un billete de lotería, más que el valor esperado de ésta. Por otro lado, en el problema 14" se prefieren pequeñas pérdidas, como ocurre en el caso de los seguros, a una gran pérdida con una probabilidad pequeña. Markowitz encontró resultados parecidos. En nuestra teoría, en el problema 14, las preferencias por la lotería implican que $\pi(.001) \mathrm{v}(5000) \mathrm{v}(5)$, así $\pi(.001)>\mathrm{v}(5) / \mathrm{v}(5000)>.001$, suponiendo que la función de valor es cóncava para las ganancias. La misma conclusión se deriva del problema 14, al preferir pagar el seguro, si se asume que la función de valor es convexa para las pérdidas.

Es importante hacer la distinción entre la sobreponderación, referida como una propiedad de los pesos de decisión, y la sobreestimación que generalmente se encuentra en la evaluación de las probabilidades de los sucesos raros. Fíjese que la sobreestimación no aparece en este contexto en el que el sujeto toma el valor dado de p. En muchas situaciones de la vida real, tanto la sobreponderación como la sobreestimación pueden producir un aumento en el impacto de los sucesos raros.

A pesar de que en $\pi(p)>p$ para probabilidades pequeñas hay evidencia para sugerir que, para todo $0<\mathrm{p}<1, \pi(\mathrm{p})+\pi(1-\mathrm{p})<1$. A esta propiedad la llamamos subcertidumbre. Se puede observar que las preferencias típicas en cualquiera de las versiones de los ejemplos de Allais (ver, por ej., los problemas 1 y 2 ) implican subcertidumbre para los valores relevantes de p. Aplicando la ecuación (1) a las preferencias prevalentes en los problemas 1 y 2 se mantiene respectivamente que:

$$
\begin{aligned}
& \mathrm{v}(2400)>\pi(.66) \vee(2400)+\pi(.33) \mathrm{v}(2500), \text { así, } \\
& {[1-\pi(.66)] \mathrm{v}(2400)>\pi(.33) \mathrm{v}(2500) \mathrm{y}} \\
& \pi(.33) \mathrm{v}(2500)>\pi(.34) \mathrm{v}(2400) ; \text { así, } \\
& 1-\pi(.66)>\pi(.34) \mathrm{o} \\
& \pi(.66)+\pi(.34)<1
\end{aligned}
$$

Aplicando el mismo análisis a los ejemplos originales de Allais se mantiene que $\pi(.89)+\pi(.11)<1$, y algunos de los datos que muestran MacCrimmon y Larsson (28) implican subcertidumbre para valores adicionales de $\mathrm{p}$.

La pendiente de $\pi$ en el intervalo $(0,1)$ se puede analizar como una medida de la sensibilidad de las preferencias por los cambios en probabilidad. La subcertidumbre implica que $\pi$ es regresiva con respecto a $\mathrm{P}$, por ej., generalmente, las preferencias son menos sensibles a las variaciones de probabilidad lo que predeciría el principio de esperanza. Así, la subcertidumbre muestra un elemento esencial de las actitudes de la gente hacia los sucesos inciertos, a saber, la suma de los pesos asociados con los suçesos complementarios es típicamente menor que el peso asociado con el evento seguro.

Recuerde que las violaciones del axioma de sustitución, tratado anteriormente, conforman la siguiente regla: $s i(x, p)$ es equivalente a $(y, p q)$ entonces $(x, p r)$ se prefiere a $(y, p q r), 0<p, p, r$

1. Por la ecuación (1), 


$$
\begin{aligned}
& \pi(\mathrm{p}) \mathrm{v}(\mathrm{x})=\pi(\mathrm{pq}) \mathrm{v}(\mathrm{y}) \text { implica } \\
& \pi(\mathrm{pr}) \mathrm{v}(\mathrm{x})<=\pi(\mathrm{pqr}) \mathrm{v}(\mathrm{y}) \text { así } \\
& {[\pi(\mathrm{pq}) / \pi(\mathrm{p})]<=[\pi(\mathrm{pqr}) / \pi(\mathrm{pr})]}
\end{aligned}
$$

Así, para una razón fija de probabilidades, la razón de los pesos de decisión correspondientes está más cerca de la unidad cuando las probabilidades son bajas que cuando son altas. Esta propiedad de $\pi$, denominada subproporcionalidad, produce restricciones considerables a la forma de $\pi$; se mantiene si y sólo si el $\log \pi$ es una función convexa del $\log \mathrm{p}$.

Es interesante destacar que la subproporcionalidad junto con la sobreponderación de probabilidades pequeñas implica que $\pi$ es subaditiva para este rango. Formalmente se puede demostrar que si $\pi(p)>p$ $y$ se mantiene la subproporcionalidad, entonces $\pi(\mathrm{rp})>\mathrm{r} \pi(\mathrm{p}), 0<\mathrm{r}<1$, dado que $\pi$ es monótona y continua en $(0.1)$.

La figura 4 presenta una función de ponderación hipotética que satisface la sobreponderación y la subaditividad para los valores pequeños de $\mathrm{p}$, al igual que la subcertidumbre y la subproporcionalidad. Estas propiedades implican que $\pi$ es relativamente llana en el intervalo abierto y cambia abruptamente cerca de los puntos-finales donde $\pi(0)=0$ y $\pi(1)=1$. Las aparentes discontinuidades de la curva de $\pi$ en los puntos finales son consistentes con la noción de que existe un límite donde los pesos de decisión pequeños hasta el cual pueden unirse a un suceso, si es que se puede dar un peso. El mismo nivel de duda aparecerá en los límites superiores sobre cualquier peso de decisión que este próximo a la unidad. Este efecto cuántico puede reflejar la distinción categórica existente entre la certidumbre y la incertidumbre. Por otro lado, la simplificación de las alternativas en la fase de preparación puede conducir al sujeto a descartar los sucesos que tienen una probabilidad extremadamente pequeña y a considerar los eventos que tienen

FIGURA 4.

Una función de ponderación bipotética.

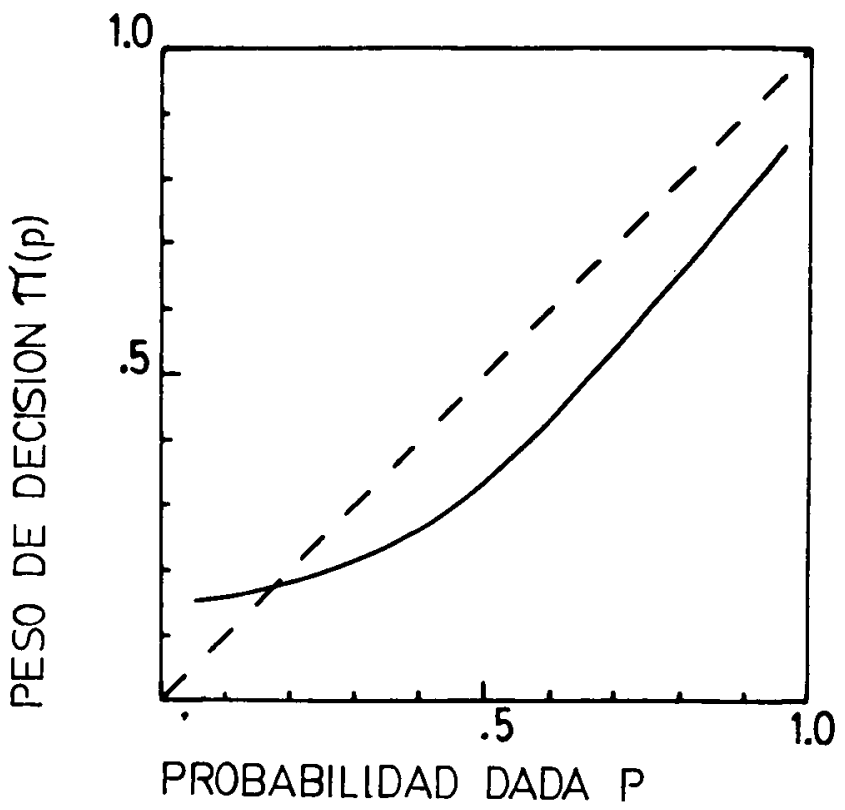


una probabilidad extremadamente alta como si fueran seguros. Debido a la existencia de limitaciones en la comprensión y evaluación de las probabilidades extıemas, los eventos que son poco probables son o bien ignorados o bien subreponderados, y la diferencia entre probabilidades altas y seguras o bien es ignorada o bien es exagerada. Consecuentemente, $\pi$ rio está bien definida cerca de los puntos finales.

El siguiente ejemplo de Zeckhauser ilustra la hipótesis de la no linealidad de $\pi$. Suponga que le ofrecen jugar a la ruleta rusa, le dan la oportunidad de quitar una de las balas de la pistola cargada. ¿Estaría dispuesto a pagar lo mismo por reducir el número de balas de 4 a 3 que por reducirlo de 1 a 0 ? La mayoría de las personas estarían dispuestas a pagar una cantidad mucho mayor con el objeto de reducir la probabilidad de muerte de $1 / 6$ a 0 que de reducirla de $4 / 6$ a $3 / 6$. Las consideraciones económicas deberían conducir a que se pague más en el último caso puesto que presumiblemente se reduce el valor del dinero debido a la probabilidad considerable de que no se viva para contarlo.

Una objeción obvia al supuesto de que $\pi(p) \neq p$ implica comparaciones entre alternativas de la forma $(\mathrm{x}, \mathrm{p} ; \mathrm{x}, \mathrm{q})$ y $\left(\mathrm{x}, \mathrm{p}^{\prime} ; \mathrm{x}, \mathrm{q}^{\prime}\right)$, donde $\mathrm{p}+\mathrm{q}=\mathrm{p}^{\prime}+\mathrm{q}^{\prime}<1$. Debido a que cualquier persona será indiferente entre las dos alternativas, se mantendrá que $\pi(\mathrm{p})+\pi(\mathrm{q})=\pi\left(\mathrm{p}^{\prime}\right)+\pi\left(\mathrm{q}^{\prime}\right)$, que implica que $\pi$ es la función de identidad. Este argumento se invalida en la presente teoría puesto que se asume que las probabilidades de resultados idénticos se combinan en la preparación dēlas alternativas. Una objeción más importante sobre la no linealidad de $\pi$ descansa en la posible violación de la dominancia. Suponga que $x>y>0, p>p^{\prime}, y$ $p^{+} q^{\prime}=p^{\prime}+q^{\circ}<1 ;$ así, $(x, p ; y, q)$ domina $\left(x, p^{\prime} ; y, q^{\prime}\right)$. Si las preferencias mantienen a la dominancia, entonces

$\mathbf{0}$

$$
\pi(\mathrm{p}) \mathrm{v}(\mathrm{x})+\pi(\mathrm{q}) \mathrm{v}(\mathrm{y})>\pi\left(\mathrm{p}^{\prime}\right) \mathrm{v}(\mathrm{x})+\pi\left(\mathrm{q}^{\prime}\right) \mathrm{v}(\mathrm{y})
$$

$$
\left[\pi(\mathrm{p})-\pi\left(\mathrm{p}^{\prime}\right)\right] /\left[\boldsymbol{\pi}\left(\mathrm{q}^{\prime}\right)-\boldsymbol{\pi}(\mathrm{q})\right]>[\mathrm{v}(\mathrm{y}) / \mathrm{v}(\mathrm{x})]
$$

Por tanto, a medida que y se aproxima a $\mathrm{x}, \boldsymbol{\pi}(\mathrm{p})-\pi(\mathrm{p})$ se aproxima a $\pi\left(q^{\prime}\right)-\pi(q)$. Puesto que $p-p^{*}=q-q^{\prime}, \pi$ debe ser esencialmente lineal, o de otra forma, se violará la dominancia.

La presente teoría evita las violaciones directas de la dominancia por el supuesto de la detección y eliminación de las alternativas dominantes antes de la evaluación de las alternativas. De todas maneras, la teoría permite violaciones indirectas de la dominancia, por ej., en las alternativas triples donde $A$ se prefiere a $B, B$ se prefiere a $C$, y $C$ domina a $A$. Ver, por ejemplo, Raiffa (34, pág. 75).

Señalemos, por último, que este tratamiento se ha aplicado a las tareas más simples de decisión donde se presentan dos alternativas. No hemos considerado en detalle tareas más complejas (por ej., apuestas) en las que se pide al sujeto que realice una apuesta de igual valor al de una alternativa dada. La simetría existente entre estas dos opciones puede dar lugar a sesgos sistemáticos. Lichtenstein y Slovic (27) formaron pares de alternativas $A$ y $B$, tales que la gente generalmente prefería $A$ a $B$, pero apostaban más por $B$ que por $A$. Este fenómeno ha sido confirmado en distintos estudios, tanto con jugadas hipotéticas como reales, por ej., Grether y Plott (20). De esta manera, no se puede suponer que generalmente el orden de preferencia de las alternativas pueda obtenerse por un procedimiento de apuestas. 
Puesto que la teoría prospectiva se ha propuesto como un modelo de elección, la inconsistencia de apuestas y elecciones implica que las medidas de los valores y de los pesos de decisión deberían basarse en las elecciones entre alternativas específicas más que en las apuestas o en otras formas la tarea. Esta restricción hace que la medida de $\mathrm{v}$ y de $\pi$ sea más difícil debido a que production tasks son más convenientes para el escalamiento que las comparaciones por pares.

\section{DISCUSION}

En esta última sección demostramos cómo la teoría prospectiva puede explicar las actitudes hacia el riesgo, discutiremos las representaciones alternativas de los problemas de elección producidos por cambios en los puntos de referencia, y plantearemos las posibles generalizaciones de nuestro enfoque.

\section{Actitudes hacia el riesgo}

Nuestra teoría explica los patrones de preferencia dominantes observados en el ejemplo de Allais (problemas 1 y 2 ) si

$$
[\pi(.33) / \pi(.34)]>[\mathrm{v}(2400) / \mathrm{v}(2500)]>[\pi(.33) / 1-\pi(.66)]
$$

Así, la violación del axioma de independencia, en este caso, se atribuye a la subcertidumbre; y, concretamente, a la desigualdad $\pi(.34)<(.66)$. Este análisis demuestra que las violaciones del tipo de Allais ocurrirán siempre que la razón $\mathrm{v}$ de los dos resultados que no sean cero esté por debajo de las razones $\pi$.

Los problemas del 3 al 8 presentan la misma estructura, por lo que es suficiente considerar un par de ellos; por ejemplo, los problemas 7 y 8. Las elecciones observadas en estos problemas se derivan de la teoría si

$$
[\pi(.001) / \pi(.002)]>[\mathrm{v}(3000) / \mathrm{v}(6000)]>[\pi(.45) / \pi(.90)]
$$

En este caso, la violación del axioma de sustitución se atribuye a la subproporcionalidad de $\pi$. Se viola la teoría de la utilidad esperada de la misma manera que se acaba de ver siempre que la razón v esté por debajo de las razones $\pi$. El mismo tipo de análisis se puede aplicar a otras violaciones del axioma de sustitución, tanto en el dominio positivo como en el negativo.

Seguidamente probaremos cómo se puede derivar de la teoría las preferencias ocurridas en el problema 9 en el que se prefería el seguro normal al seguro probabilístico, puesto que la probabilidad de las pérdidas se ponderará más. Así, si $(-\mathrm{x}, \mathrm{p}$ es indiferente a $(-\mathrm{y})$, entonces $(-\mathrm{y})$ se prefiere a $(-\mathrm{x}, \mathrm{p} / 2 ;-\mathrm{y}, \mathrm{p} / 2 ;-\mathrm{y} / 2,1-\mathrm{p})$. Para simplificar, definimos para $x>=0, f(x)=-v(-x)$. Debido a que la función de valoración es convexa para las pérdidas, $f$ es una función cóncava de $x$. Aplicando la teoría prospectiva, por la ecuación 2, queremos demostrar que

$$
\begin{aligned}
& \pi(\mathrm{p}) \mathrm{f}(\mathrm{x})=\mathrm{f}(\mathrm{y}) \text { implica } \\
& \quad \mathrm{f}(\mathrm{y}) \leq \mathrm{f}(\mathrm{y} / 2)+\pi(\mathrm{p} / 2)[\mathrm{f}(\mathrm{y})-\mathrm{f}(\mathrm{y} / 2)]+\pi(\mathrm{p} / 2) \\
& {[\mathrm{f}(\mathrm{x})-\mathrm{f}(\mathrm{y} / 2)]=} \\
& \quad=\pi(\mathrm{p} / 2) \mathrm{f}(\mathrm{x})+\pi(\mathrm{p} / 2) \mathrm{f}(\mathrm{y})+[1-2 \pi(\mathrm{p} / 2)] \mathrm{f}(\mathrm{y} / 2)
\end{aligned}
$$


Sustituyendo por $f(x)$ y por la concavidad de $f$, es suficiente con demostrar que

$$
\mathrm{f}(\mathrm{y})<=[\boldsymbol{\pi}(\mathrm{p} / 2) / \pi(\mathrm{p})] \mathrm{f}(\mathrm{y})+\boldsymbol{\pi}(\mathrm{p} / 2) \mathrm{f}(\mathrm{y})+\mathrm{f}(\mathrm{y}) / 2-\boldsymbol{\pi}(\mathrm{p} / 2) \mathrm{f}(\mathrm{y})
$$

ó

$$
\pi(\mathrm{p}) / 2<=\pi(\mathrm{p} / 2) \text { por la subaditividad de } \pi
$$

Según esta teoría, las actitudes hacia el riesgo están determinadas conjuntamente por $\mathrm{v}$ y $\pi$, y no únicamente por la función de utilidad. De todas mảneras, parece útil examinar las condiciones bajo las cuales se espera que ocurra o bien la aversión por el riesgo o bien la atracción hacia éste. Consideremos la elección entre la jugada $(\mathrm{x}, \mathrm{p})$ y su valor esperado $(\mathrm{xp})$. Si $\mathrm{x}>0$, existirá atracción por el riesgo siempre que $\boldsymbol{\pi}(\mathrm{p})>\mathrm{v}(\mathrm{px}) / \mathrm{v}(\mathrm{x})$, que es mayor que $\mathrm{p}$ si la función de valoración es cóncava para las ganancias. Así, la sobreponderación $(\pi(p)>p)$ es necesaria pero no suficiente para la atracción por el riesgo en el dominio de las ganancias. Precisamente la misma condición es necesaria pero no suficiente para la aversión al riesgo cuando $x<0$. Este análisis restringe la atracción hacia el riesgo en el dominio de las ganancias y la aversión en el dominio de las pérdidas para probabilidades pequeñas, cuando se mantiene la sobreponderación. En realidad éstas son las situaciones típicas bajo las cuales se realiza la compra de tickets de lotería y de seguros. La sobreponderación, en la teoría prospectiva, favorece tanto el juego como los seguros, mientras que la forma de $S$ de la función de valoración, tiende a inhibir dichas conductas.

Aunque la teoría prospectiva predice tanto la compra de seguros como el juego con probabilidades pequeñas, no nos sentimos satisfechos con el análisis realizado para explicar de forma totalmente adecuada estos fenómenos complejos. En realidad existe evidencia experimental proveniente tanto de estudios experimentales como de investigaciones de campo y de observaciones de la conducta económica, por ej., sobre seguros médicos y de servicios, que muestra que la compra de seguros se amplía a probabilidades de rango medio y que las bajas probabilidades de que ocurra un desastre son algunas veces ignoradas. Aún más, la evidencia muestra que pequeños cambios en la formulación de los problemas de decisión pueden producir importantes efectos sobre la atracción de un seguro. Aparte de analizar las actitudes puras hacia la incertidumbre y hacia el dinero, una teoría comprensiva de la conducta sobre los seguros deberá también tener en cuenta factores tales como el valor de la seguridad, las normas sociales de prudencia, la aversión a pagar muchas veces pequeñas cantidades de dinero, la información y la desinformación acerca de los resultados y las probabilidades; así como otros muchos. Alguno de los efectos de estas variables pueden describirse dentro del marco de esta teoría; por ej., cambios en el punto de referencia, transformaciones de la función de valoración o manipulaciones de las probabilidades y los pesos de decisión. La consideración de otros efectos podría implicar la introducción de variables o conceptos que no se han considerado en este tratamiento. 
Hasta el momento hemos definido las pérdidas y las ganancias por la cantidad de dinero que se obtenía o pagaba cuando se jugaba, y, considerábamos el punto de referencia como el status quo o como el estado actual de la persona. Aunque esto sea válido para la mayoría de los problemas de elección, existen otras situaciones donde las ganancias y las pérdidas se relacionan con las expectativas, o el nivel de aspiración difiriendo del status quo. Por ejemplo, un descuento de un impuesto inesperado por pagar un talón mensualmente se considera más una pérdida que una reducción de las ganancias. De manera parecida, una persona emprendedora que supera una bancarrota con mayor éxito que sus competidores interpretará una pequeña pérdida como una ganancia, en la relación con la inmensa pérdida que podría esperar.

En los anteriores ejemplos el punto de referencia correspondía al estado que uno esperaba lograr. Es posible que se produzca una diferencia entre el punto de referencia y el estado actual cuando se producen cambios nuevos del estado de riqueza al que uno aún no se ha adaptado. Imagínese a una persona que está inmersa en un negocio; hasta el momento, ha perdido una cantidad de 2000 y se le plantea la oportunidad de ganar 1000 seguras o de ganar 2000 o nada con una probabilidad del 50 por 100 . Si todavía no se ha adaptado a su pérdida, podrá considerar el problema como una elección entre $(-2000,50)$ y $(-1000)$ y no como una elección entre $(2000, .50)$ y $(1000)$. Como hemos visto, la primera representación induce elecciones más arriesgadas que la última.

Un cambio en el punto de referencia altera el orden de preferencia de las alternativas. Concretamente, la presente teoría implica que un. cambio en la elección de un problema, como, por ejemplo, la que se produce de una adaptación incompleta a las pérdidas recientes, aumenta, en muchas situaciones, la atracción por el riesgo. Específicamente, si una alternativa arriesgada $(x, p ;-y, 1-p)$ se acepta, entonces $(x-z, p ;-y-z, 1-p)$ se prefiere a $(-z)$ para $x, y, z>0$, con $x>z$.

Para probar esta proposición, fíjese que

$\mathrm{V}(\mathrm{x}, \mathrm{p} ; \mathrm{y}, 1-\mathrm{p})=0 \mathrm{si}$

$\pi(\mathrm{p}) \vee(\mathrm{x})=-\pi(1-\mathrm{p}) \mathrm{v}(-\mathrm{y})$

Además,

$\mathrm{V}(\mathrm{x}-\mathrm{z}, \mathrm{p} ;-\mathrm{y}-\mathrm{z}, 1-\mathrm{p})=$

$=\pi(\mathrm{p}) \mathrm{v}(\mathrm{x}-\mathrm{z})+\pi(1-\mathrm{p}) \mathrm{v}(-\mathrm{y}-\mathrm{z})>$

$>\pi(\mathrm{p}) \mathrm{v}(\mathrm{x})-\pi(\mathrm{p}) \mathrm{v}(\mathrm{z})+\pi(1-\mathrm{p}) \mathrm{v}(-\mathrm{y})+$

$+\pi(1-\mathrm{p}) \mathrm{v}(-\mathrm{z})$ por las propiedades de $\mathrm{v}$,

$=-\pi(1-\mathrm{p}) \mathrm{v}(-\mathrm{y})-\pi(\mathrm{p}) \mathrm{v}(\mathrm{z})+$

$+\pi(1-\mathrm{p}) \vee(-\mathrm{y})+\pi(1-\mathrm{p}) v(-\mathrm{z})$

por sustitución,

$=-\pi(\mathrm{p}) \mathrm{v}(\mathrm{z})+\pi(1-\mathrm{p}) \mathrm{v}(-\mathrm{z})>$

$>\mathrm{v}(-\mathrm{z})[\pi(\mathrm{p})+\pi(1-\mathrm{p})$

puesto que $\mathrm{v}(-\mathrm{z})<-\mathrm{v}(\mathrm{z})$,

$>\mathrm{v}(-\mathrm{z})$ por la subcerridumbre. 
Este análisis sugiere que una persona que no ha asumido sus pérdidas es probable que acepte jugadas que de otra manera nunca aceptaría. La observación de la tendencia a realizar apuestas mayores, conforme avanza el día de las carreras, apoya la hipótesis de que la inadaptación a las pérdidas, o que el logro de una ganancia esperada, induce a la búsqueda del riesgo. Pongamos otro ejemplo, considere una persona que está dispuesta a realizar la compra de un seguro, quizá porque en el pasado poseía uno o porque sus amigos lo tienen. El sujeto debe enfrentarse a la situación de pagar una cierta cantidad «y» con el fin de protegerse de una pérdida $\mathbf{x}$; se trata más de una elección entre $(-x+y, p ; y, 1-p)$ y $(0)$, que una elección entre $(-x, p)$ y $(-y)$. La argumentación anterior implica que es más probable que el seguro sea más atractivo de la primera forma que de la última.

Otro caso importante del cambio del punto de referencia se produce cuando el sujeto se formula el problema de decisión en términos de estados finales, tal como defiende el análisis de decisión, en lugar de hacerlo en términos de las ganancias y las pérdidas, como lo realiza la mayoría de las personas. En este caso, el punto de referencia es cero en la escala de riqueza y es probable que la función de valoración sea en todas partes cóncava. Según el presente análisis, esta formulación elimina, fundamentalmente, la atracción por el riesgo, excepto en jugadas con probabilidades pequeñas. La formulación explícita de los problemas de decisión en términos de los estados finales es, quizá, el procedimiento más efectivo para eliminar la atracción por el riesgo en el dominio de las pérdidas.

Muchas de las decisiones económicas implican transacciones en las que se tiene que pagar una cantidad de dinero a cambio de una alternativa deseable. Estos problemas son analizados por las teorías actuales de decisión como comparaciones entre el status quo y el estado de una alternativa en la que se incluye la alternativa elegida menos su coste. Por ejemplo, la decisión de pagar 10 por una jugada $(1000, .01)$ se analiza como la elección entre $(990,01 ;-10, .99)$ y $(0)$. En este análisis, la disponibilidad para pagar la alternativa positiva se iguala a la de aceptar la alternariva mixta correspondiente.

El fracaso fundamental para integrar las alternativas arriesgadas y las no arriesgadas se debe al efecto de aislamiento, que sugiere que es poco probable que la gente realice la operación de sustraer el coste de los resultados cuando deciden comprar o no una jugada. En lugar de ello, se sugiere que la gente normalmente evalúa separadamente la jugada y su coste, y decide la compra de la jugada en el caso en que el valor combinado sea positivo. Así, la jugada $(1000,01)$, por ejemplo, es prácticamente equivalente a la decisión de aceptar la jugada $(990,01 ;-10,99)$. Además, la teoría prospectiva predice que si se es indiferente entre $(x(1-p), p ;-p x, 1-p)$ y $(0)$ entonces no se pagará $p x$ para comprar la alternativa $(\mathrm{x}, \mathrm{p})$. Así, se espera que la gente muestre una mayor atracción al riesgo en la decisión de aceptar una jugada justa que en la decisión de la compra de una jugada por un precio justo. La localización del punto de referencia, y la forma en que se codifican y preparan los problemas de elección son factores críticos en el análisis de decisiones. 
La teoría prospectiva debería de ampliarse en distintas direcciones si quiere dar cuenta de un mayor rango de problemas de decisión. No parece complicada la aplicación de las ecuaciones (1) y (2) a alternativas con cualquier número de resultados. Se deberán realizar operaciones adicionales a la fase de preparación con el fin de simplificar la evaluación, en el caso de que tengamos un número considerable de resultados. Todavía tiene que investigarse la manera en la que se reducen las opciones complejas; por ejemplo, con alternativas compuestas, con el fin de simplificarlas.

A pesar de que en este artículo se han considerado, fundamentalmente, los resultados monetarios, la teoría puede aplicarse a elecciones que impliquen otros atributos, por ejemplo, calidad de vida o en número de vidas que se pueden salvar o perder como consecuencia de una decisión política. Las propiedades principales de la función de valoración que se ha propuesto para el dinero puede ser aplicada también a otros atributos. Concretamente, se espera que los resultados se codifiquen como ganancias o pérdidas con respecto a un punto neutral de referencia, y que las pérdidas se consideren mayores que las ganancias.

La teoría, también, puede aplicarse a situaciones típicas de elección, donde las probabilidades de los resultados no se explicitan. En estas situaciones, los pesos de decisión irán ligados a los sucesos particulares más que a las probabilidades dadas, pero se supone que se mantendrán las propiedades esenciales de la función de ponderación. Por ejemplo, si A y $\mathrm{B}$ son sucesos complementarios y ninguno de ellos es seguro, $\pi(\mathrm{A})+\pi(\mathrm{B})$ deberá ser menor que la unidad, lo cual es una forma natural de la subcertidumbre.

Los pesos de decisión asociados con un suceso dependerán fundamentalmente de la probabilidad que se perciba en ese suceso, que puede estar sujeta a sesgos considerables. Además a los pesos de decisión les pueden afectar otras consideraciones como la ambigüedad o la imprecisión. En realidad, el trabajo de Ellsberg y Fellner muestra que la imprecisión reduce los pesos de decisión. En consecuencia la subcertidumbre se reflejará más con las probabilidades imprecisas o vagas que con las probabilidades claras.

El presente análisis de las preferencias entre las opciones arriesgadas ha producido el desarrollo de dos líneas de estudio. La primera de ellas trata la preparación de las operaciones que determinan la percepción de las alternativas. La segunda trata los principios del juicio que dirigen la evaluación de las ganancias y de las pérdidas y la ponderación de los resultados inciertos. Estas dos líneas ofrecen un marco útil para el análisis descriptivo de la elección bajo riesgo, aunque, necesitan un mayor desarrollo.

\section{APENDICE (2)}

En este apéndice se muestra un análisis axiomático de la teoría prospectiva. Debido a que el análisisis completo es largo y tedioso vamos a apuntar esquemáticamente los pasos esenciales para mostrar las propiedades ordinales claves que son necesarias para establecer la formu- 
lación de la bilinealidad de la ecuación (1). Se aplicarán métodos parecidos en la axiomatización de la ecuación (2).

Considérese el conjunto de todas las alternativas regulares de la for$\mathrm{ma}(\mathrm{x}, \mathrm{p} ; \mathrm{y}, \mathrm{q})$ con $\mathrm{p}+\mathrm{q}<1$. La generalización para alternativas regulares con $\mathrm{p}+\mathrm{q}=1$ es sencilla. Sea $\gtrsim$ la relación de preferencias entre alternativas que es conectiva, simétrica y transitiva, y sea $\simeq$ la relación asociada a la indiferencia. Obviamente, $(\mathrm{x}, \mathrm{p} ; \mathrm{y}, \mathrm{q}) \simeq(\mathrm{y}, \mathrm{q} ; \mathrm{x}, \mathrm{p})$. Supongamos, también, como se deduce de nuestra notación, que $(\mathrm{x}, \mathrm{p} ; 0, \mathrm{q}) \simeq(\mathrm{x}, \mathrm{p} ; 0, \mathrm{r})$, $\mathrm{y}(\mathrm{x}, \mathrm{p} ; \mathrm{y}, 0) \simeq(\mathrm{x} ; \mathrm{z}, 0)$. Esto es, el resultado nulo y el suceso imposible tienen la propiedad del cero multiplicativo.

Fijese que la formulación que pretendemos (ecuación (1)) es aditiva en los pares de probabilidad-resultado. Así, la teoría de la medida conjunta aditiva puede aplicarse para la obtención de la escala $\mathrm{V}$ que preserva el orden de preferencia y un intervalo de escala «f» $y$ «g» en dos argumentos tales que

$$
V(x, p ; y, q)=f(x, p)+g(y, q)
$$

Los axiomas claves utilizados para derivar esta representación son:

Independencia: (x,p;y,q) $\gtrsim\left(x, p^{\prime} ; y^{\prime}, q^{\prime}\right)$ si $\left(x^{\prime}, p^{\prime} ; y, q\right) \gtrless\left(x^{\prime}, p^{\prime} ; y^{\prime}, q^{\prime}\right)$.

Cancelación: si $\left(x, p ; y^{\prime}, q^{\prime}\right) \gtrless\left(x^{\prime}, p^{\prime} ; y, q\right)$ y $\left(x^{\prime}, p^{\prime} ; y^{\prime \prime}, q^{\prime \prime}\right) \gtrsim\left(x^{\prime \prime}, p^{\prime \prime} ; y^{\prime}, q^{\prime}\right)$, entonces $\left(x, p ; y^{\prime \prime}, q^{\prime \prime}\right) \gtrsim\left(x^{\prime \prime}, p^{\prime \prime} ; y, q\right)$.

Continuidad: si $(\mathrm{x}, \mathrm{p} ; \mathrm{y}, \mathrm{q}) \gtrless(\mathrm{z}, \mathrm{r}) \gtrless\left(\mathrm{x}, \mathrm{p} ; \mathrm{y}^{\prime}, \mathrm{q}^{\prime}\right)$ para algún resultado z y una probabilidad $r$, entonces existe $y^{\prime \prime}, q$ " tal que

$\left(\mathrm{x}, \mathrm{p} ; \mathrm{y}^{\prime \prime}, \mathrm{q} "\right) \simeq(\mathrm{z}, \mathrm{r})$

Se ha demostrado que estas condiciones son suficientes para la formulación aditiva deseada, dado que el orden de preferencia es «Archimedean» $(8,25)$. Además, puesto que $(x, p ; y, q) \simeq(y, q ; x, p)$, $f(x, p)+g(y, q)=f(y, q)+g(x, p)$, y siendo $q=0$ se mantiene que $f=g$.

A continuación, consideremos el conjunto de todas las alternativas de la forma ( $\mathrm{x}, \mathrm{p})$ con un resultado simple \# a cero. En este caso, el modelo bilineal se reduce a $\mathrm{V}(\mathrm{x}, \mathrm{p})=\boldsymbol{\pi}(\mathrm{p}) \mathrm{v}(\mathrm{x})$. Este es el modelo multiplicativo investigado por Roskies, Krantz, Luce, Suppes y Tversky. Para construir la formulación multiplicativa se asume que el orden de los pares probabilidad-resultado satisface la independencia, la cancelación y la continuidad, y el «axioma de Archimedean». Además, asumimos la dependencia de signos (25) para asegurar la multiplicación adecuada de signos. Debe señalarse que el axioma de la continuidad usado por los susodichos autores debe estar debilitado, puesto que el factor de probabilidad sólo permite una continuidad limitada.

Combinando ambas formulaciones, la multiplicativa y la aditiva, se mantiene que

$$
\mathrm{V}(\mathrm{x}, \mathrm{p} ; \mathrm{y}, \mathrm{q})=\mathrm{f}(\boldsymbol{\pi}(\mathrm{p}) \mathrm{v}(\mathrm{x}))+\mathrm{f}(\boldsymbol{\pi}(\mathrm{q}) \mathrm{v}(\mathrm{y}))
$$

Por último, aplicamos un nuevo axioma distributivo:

$$
(\mathrm{x}, \mathrm{p} ; \mathrm{y}, \mathrm{p}) \simeq(\mathrm{z}, \mathrm{p}) \text { si }(\mathrm{x}, \mathrm{q} ; \mathrm{y}, \mathrm{q}) \simeq(\mathrm{z}, \mathrm{q})
$$

Aplicando este axioma a la formulación anterior obtenemos 
que implica

$$
f(\pi(q) v(x))+f(\pi(q) v(y))=f(\pi(q) v(z)) .
$$

Asumiendo, sin pérdida de generabilidad, que $\pi(q)<\pi(p)$ y siendo $\alpha=\pi(\mathrm{p}) \mathrm{v}(\mathrm{x}), \beta \pi(\mathrm{p}) \mathrm{v}(\mathrm{y}), \gamma=\pi(\mathrm{p}) \mathrm{v}(\mathrm{z})$ y $\theta=\pi(\mathrm{q}) / \pi(\mathrm{p})$, se mantiene que $f(\alpha)+f(\beta)=f(\gamma)$ implica $f\left(\begin{array}{ll}\theta & \alpha\end{array}\right)+f\left(\begin{array}{ll}\theta & \beta\end{array}\right)=f\left(\begin{array}{ll}\theta & \gamma\end{array}\right)$ para todo $0<\theta<1$.

Debido a que $f$ es estrictamente monótona podemos establecer $=\mathrm{f}^{-1}\left[\mathrm{f}(\alpha+\mathrm{f}(\beta)]\right.$. Así, $\theta \gamma=\theta \mathrm{f}^{-1}[\mathrm{f}(\alpha)+\mathrm{f}(\beta)]=\mathrm{f}^{-1}\left[\mathrm{f}\left(\begin{array}{ll}\theta & \alpha\end{array}\right)+\mathrm{f}\left(\begin{array}{ll}\theta & \beta\end{array}\right)\right]$

La solución a esta ecuación funcional es $\mathrm{f}(\alpha)=\mathrm{K}_{\alpha}^{\mathrm{c}}$ (1). Así, $\mathrm{V}(\mathrm{x}, \mathrm{p} ; \mathrm{y}, \mathrm{q})=\mathrm{K}[\pi(\mathrm{p}) \mathrm{v}(\mathrm{x})]^{\mathrm{c}}+\mathrm{K}[\boldsymbol{\pi}(\mathrm{q}) \mathrm{v}(\mathrm{y})]^{c}$, para algún $\mathrm{K}, \mathrm{c}>0$. La forma bilineal se obtiene redefiniendo las escalas $\pi, v, y \mathrm{~V}$ es tal que absorbe las constantes $\mathrm{K}$ y $\mathrm{c}$.

\section{Referencias}

ACZEL, J. Lectures on Functional Equations and Their Applications. Nueva York: Academic Press, 1966. Allais, M. «Le Comportement de l'Homme Rationnnel devant le Risque, Critique des Postulats et Axiomes de l'Ecole Americaine», Econometrica, 1953, 21, 503-546.

ANDERSON, N. H., y ShanteAU, J. C. "Information Integration in Risky Decision Making», Journal of Experimental Psychology, 1970, 84, 441-451.

ARROW, K. J. Essays in the Theory of Risk-Bearing. Chicago, Markham, 1971.

BARNES, J. D., y REINMUTH, J. E. «Comparing Imputed and Actual Utility Functions in a Competitive Bidding Setting», Decision Sciences, 1976, 7, 801-812.

COOMBS, C. H. «Portfolio Theory and the Measurement of Risk», en M. F. Kaplan y S. Schwartz (Eds.): Human Judgment and Decision Processes. Nueva York: Academic Pres, 1975, 63-85.

DAVIDSON, D.; SUPPES, P., y SIEGEL, S. Decision-making: An Experimental Approach. Stanford University Press, 1957.

Debreu, G. "Topological Methods in Cardinal Utility Theory», K. J. Arrow, S. Karlin y P. Suppes en Matbematical Methods in the Social Scien Stanford: Stanford University Press, 1960, 16-26.

EDWARDS, W. "Subjective Probabilities Inferred from Decisions". Psychological Review, 1962, 69, 109-135.

ELLSBERG, D. «Risk, Ambiguity and the Savage Axioms». Quarterly Journal of Economics, 1961, 75, 643-669.

FeLLNER, W. «Distortion of Subjective Probabilities as a Reaction to Uncertainty». Quarterly Journal of Economics, 1961, 75, 670-690.

FELLNER, W. Probability and Profit-A Study of Economic Behavior Along Bayesian Lines. Homewood, Illinois: Richard D. Irwin, 1965.

FishbURN, P. C. «Mean-Risk Analysis with Risk Associated with Below-Target Returns». American Economic Review, 1977, 67, 116-126.

Fishburn, P. C., y Kochenberger, G. A.: «Two-Piece von Neumann-Morgenstern Utility Functions» (en prensa).

Friedman, L., y Savage, L. J. «The Utility Analysis of Choices Involving Risks». Journal of Political Economy, 1948, 56, 279-304.

Fuchs, V. R. «From Bismark to Woodcock: The "Irrational" Pursuit of National Health Insurance». Journal of Law and Economics, 1976, 19, 347-359.

Galanter, E., y Puiner, P. «Cross-Modality Matching of Money Against Other Continua», en H. r. Moskowitz et al. Sensation and Measurement, Dordrecht, Holland: Reidel, 1974, 65-76.

Grayson, C. J. Decisions under Uncertainty: Drilling Decisions by Oil and Gas Operators. Cambridge, Massachusetts: Graduate School of Business, Harvard University, 1960.

GREEN, P. E. «Risk Attitudes and Chemical Investment Decisions». Chemical Engineering Progress, 1963, $59,35-40$.

Grether, D. M. y Plott, C. R. «Economic Theory of Choice and the Preference Reversal Phenomenon», American Economic Review (en prensa).

Halter, A. N. y Denn, G. W. Decisions under Uncertainty. Cincinnati: South Western Publishing Co., 1971.

HANSSON, B. «The Appropriateness of the Expected Utility Model». Erkenntnis, 1975, 9, 175-194.

HELSON, H. Adaptation-Level Theory. Nueva York: Harper, 1964.

KeENEY, R. L. y RAIfFA, H. Decisions with Multiple Objectives: Preferences and Value Tradeoffs. Nueva York: Wiley, 1976. 
KRANTZ, D. H.; LuCE, D. R.; SUPPES, P. y TVerSkY, A. Foundations of Measurement. Nueva York: Academic Press, 1971.

KunReuther, H.; Ginsberg, R.; Miller, L.; Sagi, P.; Slovic, P.; Borkan, B., y Katz, N. Disaster Insurance Protection: Public Policy Lessons. Nueva York: Wiley, 1978.

Lichtenstein, S., y Slovic, P. «Reversal of Preference Between Bids and Choices in Gambling Decisions». Journal of Experimental Psychology, 1971, 89, 46-55.

MacCrimmon, K. R. y LARSSON, S. «Utility Theory: Axioms versus Paradoxes», M. Allais y O. Hagen (Eds.): Expected Utility Hypothesis and the Allais Paradox. M. Allais en preparación. (En Theory and Decision).

MARKowITZ, H.: "The Utility of Wealth». Journal of Political Economy, 1952, 60, 151-158.

Markowitz, H. Portfolio Selection. Nueva York: Wiley, 1959.

MCGlothlin, W. H. «Stability of Choices among Uncertain Alternatives». American Journal of Psycho$\log y, 1956,69,604-615$.

MOSTElleR, F., y NOGEe, P. «An Experimental Measurement of Utility». Journal of Political Economy, 1951, 59, 371-404.

PRATT, J. W. «Risk Aversion in the Small and in the Large». Econometrica, 1964, 32, 122-136.

RAIFFA, H. Decision Analysis: Introductory Lectures on Choices Under Uncertainty. Reading, Massachusetts: Addison-Wesley, 1968.

RoskiEs, R. «A Measurement Axiomatization for an Essentially Multiplicative Representation of Two Factors». Journal of Mathematical Psychology, 1965, 2, 266-276.

SaVAGE, L. J. The Foundations of Statistics. Nueva York: Wiley, 1954.

Slovic, P.; FischHofF, B.; Lichtenstein, S.; CoRrigan, B:, y CoOMBS, B.: «Preference for Insuring Against Probable Small Losses: Insurance Implications». Journal of Risk and Insurance, 1977, 44, 237-258.

Slovic, P., y Tversky, A. «Who Accepts Savage's Axiom?». Bebavioral Science, 1974, 19, 368-373.

SPETZLER, C. S. «The Development of Corporate Risk Policy for Capital Investment Decisions». IEEE Transactions on Systems Science and Cybernetics, SSC-4, 1968, 279-300.

Swalm, R. O. «Utility Theory-Insights into Risk Taking». Harvard Business Review, 1966, 44, 123-136.

ToBIN, J. «Liquidity Preferences as Behavior Towards Risk». Review of Economic Studies, 1958, 26, 65-86.

TVERSKY, A. «Additivity, Utility, and Subjective Probability». Journal of Mathematical Psychology, 1967, 4, 175-201.

TVERSKY, A. «Intransitivity of Preferences». Psychological Review, 1969, 76, 31-48.

TVERSKY, «Elimination by Aspects: A Theory of Choice». Psychological Review, 1972, 79, 281-299.

TVERSKY, A., y KaHNEMAN, D. «Judgment under Uncertainty: Heuristics and Biases». Science, 1974, 185, 1124-1131.

VAN DAM, C. «Another Look at Inconsistency in Financial Decision-Making», presentado en el Seminar on Recent Research in Finance and Monetary Economics, Cergy-Pontoise, marzo de 1975.

von Neumann, J., y MoRgenstern, O. Theory of Games and Economic Behavior. Princeton: Princeton University Press, 1944.

Williams, A. C. «Attitudes toward Speculative Risks as an Indicator of Attitudes toward Pure Risks». Journal of Risk and Insurance, 1966, 33, 577-586. 